HIP-2009-22/TH

DO-TH 09/20

\title{
Implications of different supersymmetry breaking patterns for the spectrum and decay of neutralinos and charginos
}

\author{
Katri Huitu ${ }^{1 *}$, Jari Laamanen ${ }^{2,3} \dagger$, P. N. Pandita ${ }^{4} \ddagger$, and Paavo Tiitola ${ }^{1} \S$ \\ 1 Department of Physics, and Helsinki Institute of Physics, \\ P. O. Box 64, FIN-00014 University of Helsinki, Finland \\ 2 Theoretical High Energy Physics, Radboud University Nijmegen, \\ P.O. Box 9010, NL-6500 GL Nijmegen, The Netherlands \\ 3 Institut für Physik, Technische Universität Dortmund, D-44221 Dortmund, Germany and \\ 4 Department of Physics, North Eastern Hill University, Shillong 793 022, India
}

\begin{abstract}
We consider different patterns of supersymmetry breaking gaugino masses, and implications of these patterns for the phenomenology of neutralinos and charginos in models of low energy supersymmetry. We outline a general procedure for obtaining approximate values for the neutralino masses relevant for our analysis, and describe the constraints on the gaugino mass parameters which follow from the present experimental limits on the mass of the lightest chargino. We evaluate an upper bound on the mass of the lightest neutralino that follows from the structure of the mass matrix in different models for the gaugino mass parameters. Using the experimental lower limit for the chargino mass, we examine the lower bound for the neutralino masses. Using a sum rule for the squared masses of charginos and neutralinos, we discuss how to distinguish between different models for the supersymmetry breaking scenarios. We discuss in detail the decay modes of neutralinos and charginos in different models of supersymmetry breaking. Our analysis shows that by measuring the masses and decay properties of the neutralinos, one can distinguish between different patterns of supersymmetry breaking in the gaugino sector. We then compare the dark matter characteristics that arise in different models of supersymmetry breaking.

PACS numbers: $12.60 . \mathrm{Jv}, 14.80 . \mathrm{Nb}, 14.80 . \mathrm{Ly}$

Keywords: Gaugino masses, supersymmetry breaking
\end{abstract}

\section{INTRODUCTION}

Supersymmetry is at present a leading candidate for physics beyond the standard model (SM). In supersymmetric models the Higgs sector of the standard model, which is crucial for its internal consistency, is technically natural, which makes these models very appealing. Since in nature there are no supersymmetric particles with the same mass as ordinary particles, supersymmetry must be a broken symmetry at low energies. The specific mechanism which breaks supersymmetry is important in determining the masses of superpartners of the SM particles, and, hence, the experimental signatures of supersymmetry. It is widely expected that at least some of the superpartners will be produced at the Large Hadron Collider (LHC), which has started its operations. However, most of the supersymmetric particles that are likely to be produced at the LHC will not be detected as such, since they will eventually decay into the lightest supersymmetric particle (LSP), which is stable as long as the R-parity $\left(R_{p}\right)$ is conserved. Thus, the experimental study of supersymmetry involves the study of cascade decays of the supersymmetric particles to the LSP, and the subsequent reconstruction of the decay chains. The LSP in a large class of supersymmetry breaking models is the lightest neutralino, which has, therefore, been a subject of intense study for a long time [1] 8$]$. A stable lightest neutralino is also an excellent candidate for dark matter [9]. In view of the possible production of supersymmetric particles and their subsequent decay into the lightest neutralino at the LHC, the properties of the lightest neutralino, and also those of heavier neutralinos and charginos, which often appear in the cascade decays, are of considerable importance. In particular a detailed study of the lightest neutralino, especially the predictions for its mass, are of great importance for the supersymmetric phenomenology.

\footnotetext{
* Electronic address: katri.huitu@helsinki.fi

$\dagger$ Electronic address: j.laamanen@science.ru.nl

Electronic address: ppandita@nehu.ac.in

$\S$ Electronic address: paavo.tiitola@helsinki.fi
} 
In the minimal supersymmetric extension of the standard model (MSSM) at least two Higgs doublets $H_{1}$ and $H_{2}$ with hypercharge $(Y)$ having values -1 and +1 , respectively, are required to generate masses for all the SM fermions and gauge bosons, and to cancel triangle anomalies. By the minimal supersymmetric extension of the SM we here mean the model with minimal particle content and the standard model gauge group. The fermionic partners of these Higgs doublets mix with the fermionic partners of the gauge bosons to produce four neutralino states $\tilde{\chi}_{i}^{0}, i=1,2,3,4$, and two chargino states $\tilde{\chi}_{i}^{ \pm}, i=1,2$. In extended supersymmetric models, there can be extra neutralino states [3, 4].

The masses of the neutralinos and charginos depend, besides other model parameters, on the soft supersymmetry breaking gaugino masses corresponding to the $S U(2)_{L}$ and $U(1)_{Y}$ gauge groups. At present there are several models of supersymmetry breaking. The model of supersymmetry breaking that has been studied most extensively is the gravity mediated [10] supersymmetry breaking model. In this class of models, supersymmetry is assumed to be broken in a hidden sector by fields which interact with the SM particles and their superpartners (the visible sector) via only the gravitational interactions. Whereas this mechanism of supersymmetry breaking is simple and appealing, it suffers from the supersymmetric flavor problem. On the other hand, in a different class of models [11], supersymmetry is broken in a hidden sector and transmitted to the visible sector via SM gauge interactions of messenger particles. This mechanism of supersymmetry breaking, the gauge mediated supersymmetry breaking, provides an appealing solution to the supersymmetric flavor problem. Both, the gravity mediated and gauge mediated supersymmetry breaking (GMSB) models, have their distinct experimental signatures. In the GMSB models, the lightest supersymmetric particle is gravitino instead of a neutralino.

The soft supersymmetry breaking terms in the two breaking mechanisms described above have contributions originating from the super-Weyl anomaly via loop effects. If gravity and gauge mediation of supersymmetry breaking are somehow suppressed, the anomaly mediated contributions can dominate, as may happen, e.g., in brane models [12]. If this happens, then this mechanism of supersymmetry breaking is referred to as anomaly mediated supersymmetry breaking (AMSB). Anomaly mediation is a predictive framework for supersymmetry breaking in which the breaking of scale invariance mediates between hidden and visible sectors.

There is another simple pattern of supersymmetry breaking, which is a hybrid between anomaly mediated supersymmetry breaking and mSUGRA pattern, that arises from the mirage (or mixed modulus) mediation of supersymmetry breaking, which has low energy values for masses quite distinct from either of the two. Mirage mediation is naturally realized in KKLT-type moduli stabilization [13] and its generalizations, a well known example being KKLT moduli stabilization in type IIB string theory [14].

The soft gaugino masses provide a handle for identifying the type of supersymmetry breaking [15 [17] in the gaugino sector. In [15] explicit examples of models of each of the patterns mentioned above were discussed. The possibility to detect the gaugino mass nonuniversality at the LHC was studied in [16]. It is because of the distinctive patterns of gaugino masses that one is tempted to believe that neutralinos and charginos are a key in understanding the supersymmetry breaking mechanism.

In this paper we consider the phenomenology of neutralinos and charginos in different models for the soft supersymmetry breaking gaugino masses. This includes the gravity mediated supersymmetry breaking, the anomaly mediated supersymmetry breaking, and the mirage mediation of supersymmetry breaking. In Section II we recall the essential features of the neutralino mixing and the resulting mass matrix, and the constraints on the parameters of the mass matrix that follow from the experimental lower bound on the mass of the lightest chargino. We then discuss the patterns of the gaugino masses that arise in the different patterns of supersymmetry breaking in the gaugino sector, and compute the neutralino mass spectrum and discuss the distinguishing features of the spectrum in different patterns of supersymmetry breaking gaugino masses. In Section III we discuss a general upper bound on the mass of the lightest neutralino, and evaluate this bound for different models for the soft gaugino masses. Here we also discuss an upper bound on the lightest neutralino mass that follows from radiative electroweak symmetry breaking, which determines the absolute value of the Higgs(ino) mass parameter $\mu$. In Section IV we discuss and evaluate a sum rule involving the squared masses of the charginos and neutralinos, and describe how this sum rule can be used to distinguish between different models of supersymmetry breaking gaugino masses. In Section V we study the two-body decays of charginos and neutralinos in different models, and discuss how the branching ratios for these decays can be used to distinguish between the underlying pattern of supersymmetry breaking gaugino masses. In Section VI we compute and compare the relic abundance of the lightest neutralinos in different models assuming that the lightest neutralino is the lightest supersymmetric particle. We discuss the constraints imposed on the parameter space by the precise limits on the relic density obtained by the Wilkinson Microwave Anisotropy Probe (WMAP) satellite [18]. Finally, in Section VII we summarize our conclusions. 


\section{CHARGINO AND NEUTRALINO MASSES AND GAUGINO MASS PATTERNS}

In this section we will describe constraints on the parameters of the neutralino mass matrix which follow from the present experimental limits on the mass of the lightest chargino. We will then discuss the patterns for the gaugino mass parameters that arise in different supersymmetry breaking scenarios, and the resulting consequences for the mass of the lightest neutralino. For completeness, we have summarized the neutralino and chargino mass matrices [19] in the Appendix, and outlined the procedure to obtain approximate eigenvalues for the neutralino mass matrix.

\section{A. Experimental Constraints}

Collider experiments have searched for the supersymmetric partners of the standard model particles. No supersymmetric partners of the SM particles have been found in these experiments. At present only lower limits on their masses have have been obtained. In particular, the search for the lightest chargino state at LEP have yielded lower limits on its mass [20]. The lower limit depends on the spectrum of the model [21]. Assuming that $m_{0}$ is large, the limit on the lightest chargino mass following from nonobservation of chargino pair production in $e^{+} e^{-}$collisions is

$$
M_{\tilde{\chi}_{1}^{ \pm}} \gtrsim 103 \mathrm{GeV}
$$

The bound depends on the sneutrino mass. For a sneutrino mass below $200 \mathrm{GeV}$, the bound becomes weaker, since the production of a chargino pair becomes more rare due to the negative interference between $\gamma$ or $Z$ in the $s$-channel and $\tilde{\nu}$ in the $t$-channel. In the models we consider, $m_{\tilde{\nu}}$ is close to $m_{0}$. When $m_{\tilde{\nu}}<200 \mathrm{GeV}$, but $m_{\tilde{\nu}}>m_{\tilde{\chi}} \pm$, the limit becomes [21]

$$
M_{\tilde{\chi}_{1}^{ \pm}} \gtrsim 85 \mathrm{GeV}
$$

For the parameters of the chargino mass matrix the limit (2.1) implies an approximate lower limit [22, 23]

$$
M_{2}, \quad \mu \gtrsim 100 \mathrm{GeV} \text {. }
$$

The limits Eq. (2.3) on the parameters $M_{2}$ and $\mu$ are found from scanning over the MSSM parameter space and are thus model independent.

\section{B. Gaugino Mass Patterns}

Having constrained the parameters $M_{2}$ and $\mu$, which enter the chargino as well as the neutralino mass matrix, we now turn to the theoretical models for the supersymmetry breaking gaugino mass parameters $M_{1}, M_{2}$, and $M_{3}$. Theoretically, a simple set of patterns has emerged for these SUSY breaking parameters, which can be described as follows.

\section{Gravity mediated supersymmetry breaking}

The first pattern, which has been the object of extensive studies, is the one which arises in the gravity mediated supersymmetry breaking models [10]. We recall that the soft gaugino masses $M_{i}$ and the gauge couplings $g_{i}$ satisfy the renormalization group equations (RGE's) $\left(\left|M_{3}\right| \equiv M_{\tilde{g}}\right.$, the gluino mass)

$$
\begin{aligned}
16 \pi^{2} \frac{d M_{i}}{d t} & =2 b_{i} M_{i} g_{i}^{2}, & & b_{i}=\left(\frac{33}{5}, 1,-3\right), \\
16 \pi^{2} \frac{d g_{i}}{d t} & =b_{i} g_{i}^{3}, & t & =\ln \left(\mu / \mu_{0}\right),
\end{aligned}
$$

at the leading order, where $i=1,2,3$ refer to the $U(1)_{Y}, S U(2)_{L}$ and the $S U(3)$ gauge groups, respectively, and $\mu$ is the renormalization scale with $\mu_{0}$ as a reference scale. Furthermore, $g_{1}=\sqrt{\frac{5}{3}} g^{\prime}, g_{2}=g$, and $g_{3}$ is the $S U(3)_{C}$ gauge coupling. In the minimal supersymmetric standard model with gravity mediated supersymmetry breaking and with a universal gaugino mass $m_{1 / 2}$ at the grand unified scale (GUT), usually referred to as mSUGRA scenario, we have the boundary conditions $\left(\alpha_{i}=g_{i}^{2} / 4 \pi, i=1,2,3\right)$

$$
M_{1}=M_{2}=M_{3}=m_{1 / 2},
$$




$$
\alpha_{1}=\alpha_{2}=\alpha_{3}=\alpha_{G}
$$

at the GUT scale $M_{G}$. The RGE's (2.4) and (2.5) imply that the soft supersymmetry breaking gaugino masses scale like gauge couplings:

$$
\frac{M_{1}\left(M_{Z}\right)}{\alpha_{1}\left(M_{Z}\right)}=\frac{M_{2}\left(M_{Z}\right)}{\alpha_{2}\left(M_{Z}\right)}=\frac{M_{3}\left(M_{Z}\right)}{\alpha_{3}\left(M_{Z}\right)}=\frac{m_{1 / 2}}{\alpha_{G}},
$$

which implies that $M_{i} / g_{i}^{2}$ does not run at the one-loop level. Although in the context of the gravity mediated supersymmetry breaking models arbitrary soft gaugino masses are possible, we shall here consider the mSUGRA realisation (2.6) of the gravity mediated supersymmetry breaking scheme. The relation (2.8) reduces the three gaugino mass parameters to one, which we take to be the gluino mass $M_{\tilde{g}}$. The other gaugino mass parameters are then determined through

$$
\begin{aligned}
& M_{1}\left(M_{Z}\right)=\frac{5 \alpha}{3 \alpha_{3} \cos ^{2} \theta_{W}} M_{\tilde{g}} \simeq 0.14 M_{\tilde{g}} \\
& M_{2}\left(M_{Z}\right)=\frac{\alpha}{\alpha_{3} \sin ^{2} \theta_{W}} M_{\tilde{g}} \simeq 0.28 M_{\tilde{g}}
\end{aligned}
$$

where we have used the value of various couplings at the $Z^{0}$ mass

$$
\alpha^{-1}\left(M_{Z}\right)=127.9, \quad \sin ^{2} \theta_{W}=0.23, \quad \alpha_{3}\left(M_{Z}\right)=0.12 .
$$

For the gaugino mass parameters this leads to the ratio

$$
M_{1}: M_{2}: M_{3} \simeq 1: 2: 7.1 \text {. }
$$

This pattern is typical of any scheme obeying Eqs. (2.4) and (2.6). Note that the masses above are the running masses evaluated at the electroweak scale, $M_{Z}$. This discussion of the gaugino mass parameters is valid at tree level. When the radiative corrections are included, the ratio for these parameters in mSUGRA is modified to

$$
M_{1}: M_{2}: M_{3} \simeq 1: 1.9: 6.2 .
$$

Using the ratio (2.13) and the lower limit (2.3), we have the constraint

$$
M_{1} \gtrsim 50 \mathrm{GeV}
$$

in the gravity mediated supersymmetry breaking models.

It is important to point out here that whereas the mechanism of gravity mediated supersymmetry breaking is simple and appealing, it suffers from the supersymmetric flavor problem. On the other hand in a different class of models, supersymmetry is broken in the hidden sector and transmitted to the visible sector via Standard Model gauge interactions of messanger particles. This mechanism of supersymmetry breaking, the gauge mediated supersymmetry breaking [11], provides an appealing solution to supersymmetric flavor problem. In order to maintain the successful gauge coupling unification of the minimal supersymmetric standard model, it is usually assumed that the messanger particles form a full GUT multiplet, in which case the resulting gaugino masses follow the mSUGRA pattern (2.12) as a consequence of the assumption of gauge coupling unification at the GUT scale. However, there are more general gauge mediated supersymmetry breaking models [24] which allow any hierarchy of gaugino masses. However, we will confine here to the minimal models in which the gaugino masses follow the mSUGRA pattern (2.12).

\section{Anomaly mediated supersymmetry breaking}

The second pattern of gaugino masses, which is distinct from the mSUGRA pattern and emerges under theoretical assumptions that are appealing, arises in the anomaly mediated supersymmetry breaking models. Since the soft supersymmetry breaking parameters are determined by the breaking of the scale invariance, they can be written in terms of the beta functions and anomalous dimensions in the form of relations which hold at all energies. In the minimal supersymmetric standard model (MSSM), the pure anomaly mediated contributions to the soft supersymmetry breaking parameters $M_{\lambda}$ (gaugino mass), $m_{i}^{2}$ (soft scalar mass squared), and $A_{y}$ (the trilinear supersymmetry breaking coupling, where $y$ refers to the Yukawa coupling) can be written as

$$
M_{\lambda}=\frac{\beta_{g}}{g} m_{3 / 2}
$$




$$
\begin{aligned}
m_{i}^{2} & =-\frac{1}{4}\left(\frac{\partial \gamma_{i}}{\partial g} \beta_{g}+\frac{\partial \gamma_{i}}{\partial y} \beta_{y}\right) m_{3 / 2}^{2}, \\
A_{y} & =-\frac{\beta_{y}}{y} m_{3 / 2}
\end{aligned}
$$

where $m_{3 / 2}$ is the gravitino mass, $\beta$ 's are the relevant $\beta$ functions, and $\gamma$ 's are the anomalous dimensions of the corresponding chiral superfields. An immediate consequence of these relations is that supersymmetry breaking terms are completely insensitive to physics in the ultraviolet. The degrees of freedom that are excited at a given energy determine the anomalous dimensions and beta functions, thus completely specifying the soft supersymmetry breaking parameters at that energy. We note that the gaugino masses are proportional to their corresponding gauge group $\beta$ functions with the lightest supersymmetric particle being mainly a wino. However, it turns out that the pure scalar mass-squared anomaly contribution for sleptons is negative [25]. There are a number of proposals for resolving this problem of tachyonic slepton masses [26-30], but some of the solutions may spoil the most attractive feature of the anomaly mediated models, i.e., the renormalization group (RG) invariance of the soft terms and the consequent ultraviolet insensitivity of the mass spectrum. A simple phenomenologically attractive way of parametrizing the nonanomaly mediated contributions to the slepton masses, so as to cure their tachyonic spectrum, is to add a common mass parameter $m_{0}$ to all the squared scalar masses [31], assuming that such an addition does not reintroduce the supersymmetric flavor problem. Such an addition of a nonanomaly mediated term destroys the attractive feature of the RG invariance of soft masses. However, the RG evolution of the resulting model, nevertheless, inherits some of the simplicity of the pure anomaly mediated relations.

There are several alternative ways to generate these extra contributions to the soft squared masses in the anomaly mediated supersymmetry breaking scheme. In particular there are models of supersymmetry breaking mediated through a small extra dimension, where SM matter multiplets and a supersymmetry breaking hidden sector are confined to opposite four-dimensional boundaries while gauge multiplets lie in the bulk. We note that in this scenario the soft gaugino mass terms are due to the anomaly mediated supersymmetry breaking, and, therefore, are governed by (2.15). On the other hand, scalar masses get contributions from both anomaly mediation and a tiny hard breaking of supersymmetry by operators on the hidden sector boundary. These operators contribute to scalar masses at one loop and this contribution is dominant, thereby making all squared scalar masses positive. The gaugino spectrum is unaltered, and the model resembles an anomaly mediated supersymmetry breaking model with nonuniversal scalar masses [32].

Using Eq. (2.15), we then have the following pattern for the ratio of the gaugino masses at tree level:

$$
M_{1}: M_{2}: M_{3} \simeq 3.3: 1: 9,
$$

which, after radiative corrections (assuming $m_{3 / 2}=40 \mathrm{TeV}$ ) are included, becomes

$$
M_{1}: M_{2}: M_{3} \simeq 2.8: 1: 7.1
$$

in the minimal supersymmetric standard model with anomaly mediated supersymmetry breaking. Schemes in which this pattern is realized require a strict separation of hidden sector that breaks SUSY from the visible sector of the MSSM. This implies a strong sequestering, and requires that all supersymmetry breaking fields are sequestered from the visible sector. Nevertheless, it may be achieved in certain class of theories with extra dimensions or a conformal field theory sector.

Using (2.3), and the anomaly pattern of the gaugino masses (2.19), we have

$$
M_{1} \gtrsim 280 \mathrm{GeV} \text {. }
$$

This is to be contrasted with the corresponding result (2.14) for the gravity mediated supersymmetry breaking.

\section{Mirage mediated supersymmetry breaking}

There is a third simple gaugino mass pattern that arises from the mirage (or mixed modulus) mediation supersymmetry breaking, which is a hybrid between anomaly mediated supersymmetry breaking and mSUGRA pattern, and has low energy values for masses quite distinct from either of the two. Mirage mediation is naturally realized in KKLT-type moduli stabilization and its generalizations, a well known example being KKLT moduli stabilization in type IIB string theory [14]. Phenomenology and cosmology of mirage mediation have been studied in 33 - 41 . Signatures of the scenario at LHC and the spectrum of neutralino mass in particular have been studied in [15, 42]. 
The boundary conditions for the soft supersymmetry breaking terms that produce the mirage mediation scheme can be written as [4]

$$
\begin{aligned}
M_{a} & =M_{0}\left[1+\frac{\ln \left(\bar{M}_{P l} / m_{3 / 2}\right)}{16 \pi^{2}} b_{a} g_{a}^{2} \alpha\right] \\
A_{i j k} & =M_{0}\left[\left(a_{i}+a_{j}+a_{k}\right)-\frac{\ln \left(\bar{M}_{P l} / m_{3 / 2}\right)}{16 \pi^{2}}\left(\gamma_{i}+\gamma_{j}+\gamma_{k}\right) \alpha\right] \\
m_{i}^{2} & =M_{0}^{2}\left[c_{i}-\frac{\ln \left(\bar{M}_{P l} / m_{3 / 2}\right)}{16 \pi^{2}} \theta_{i} \alpha-\left(\frac{\ln \left(\bar{M}_{P l} / m_{3 / 2}\right)}{16 \pi^{2}}\right)^{2} \dot{\gamma}_{i} \alpha^{2}\right]
\end{aligned}
$$

where $M_{0} \sim 1 \mathrm{TeV}$ is a mass parameter characterizing the moduli mediation, $\bar{M}_{P l}$ is the reduced Planck mass, $g_{a}$ are the gauge couplings and $b_{\alpha}$ the corresponding one-loop beta function coefficients, $\gamma_{i}$ are the anomalous dimensions, and $\alpha=m_{3 / 2} /\left[M_{0} \ln \left(\bar{M}_{P l} / m_{3 / 2}\right)\right]=\mathcal{O}(1)$ is a parameter representing the ratio of anomaly mediation to moduli mediation, and

$$
\begin{aligned}
\theta_{i} & =4 \sum_{a} g_{a}^{2} C_{2}^{a}\left(\phi_{i}\right)-\sum_{j k}\left|y_{i j k}\right|^{2}\left(a_{i}+a_{j}+a_{k}\right), \\
\dot{\gamma}_{i} & =8 \pi^{2} \frac{d \gamma_{i}}{d \log \mu},
\end{aligned}
$$

where $C_{2}^{a}\left(\phi_{i}\right)$ is the quadratic Casimir operator for the gauge group with gauge coupling $g_{a}$, and has a value $\left(N^{2}-\right.$ 1) $/(2 N)$ for the $S U(N)$ gauge group.

Thus the generic mirage mediation is parametrized by

$$
M_{0}, \alpha, a_{i}, c_{i}=1-n_{i}, \tan \beta .
$$

The parameter values $c_{i}=a_{i}=1$ and $\alpha=1$ correspond to the minimal KKLT compactification of type IIB theory with modular weight $n_{i}=0$, but other parameter values are also possible for different scenarios, for example choice of $\alpha=2$ with $a_{H_{U}}=c_{H_{U}}=0$ and $a_{U_{3}}+a_{Q_{3}}=c_{U_{3}}+c_{Q_{3}}=0$ can possibly alleviate the fine tuning problem for the electroweak symmetry breaking [44]. In our studies we have used the values $c_{i}=a_{i}=1$. At low energies, the gaugino masses in mirage mediation can be written as

$$
\frac{M_{a}(\mu)}{g_{a}^{2}(\mu)}=\left(1+\frac{\ln \left(\bar{M}_{P l} / m_{3 / 2}\right)}{16 \pi^{2}} g_{G U T}^{2} b_{a} \alpha\right) \frac{M_{0}}{g_{G U T}^{2}} .
$$

This leads to a unification of the soft gaugino masses at the mirage messenger scale [45]

$$
M_{\mathrm{mir}}=M_{G U T}\left(\frac{m_{3 / 2}}{\bar{M}_{P l}}\right)^{\alpha / 2}
$$

which is lower than GUT scale for positive values of $\alpha$. For $g_{G U T}^{2} \simeq 1 / 2$ the resulting low energy values yield the mirage mass pattern

$$
M_{1}: M_{2}: M_{3} \simeq(1+0.66 \alpha):(2+0.2 \alpha):(6-1.8 \alpha) .
$$

When the radiative corrections are included for the supersymmetry breaking gaugino masses, we obtain

$$
\begin{array}{cc}
M_{1}: M_{2}: M_{3} \simeq 1: 1.5: 2.1 & \text { for } \alpha=1, \\
M_{1}: M_{2}: M_{3} \simeq 1: 1.2: 0.92 & \text { for } \alpha=2 .
\end{array}
$$

where we have used the value $M_{0}=1 \mathrm{TeV}$. Thus, for the mirage mediation, we find

$$
\begin{array}{ll}
M_{1} \gtrsim 67 \mathrm{GeV} & \text { for } \alpha=1, \\
M_{1} \gtrsim 83 \mathrm{GeV} & \text { for } \alpha=2 .
\end{array}
$$




\begin{tabular}{|l|c|c|c|c|}
\hline Parameters & mSUGRA & AMSB & Mirage $\alpha=1$ & Mirage $\alpha=2$ \\
\hline $\tan \beta=5, m_{0}=200 \mathrm{GeV}$ & $(58,105,250,277)$ & $(85,245,505,518)$ & & \\
& $(103,278)$ & $(85,518)$ & & \\
$\tan \beta=20, m_{0}=200 \mathrm{GeV}$ & $(58,104,229,253)$ & $(85,237,474,482)$ & & \\
& $(103,253)$ & $(85,484)$ & & \\
$\tan \beta=5, m_{0}=1 \mathrm{TeV}$ & $(55,103,346,363)$ & $(102,286,629,638)$ & $(72,85,165,176)$ & $(163,186,473,489)$ \\
& $(103,365)$ & $(103,640)$ & $(85,184)$ & $(174,479)$ \\
$\tan \beta=20, m_{0}=1 \mathrm{TeV}$ & $(58,104,211,240)$ & $(103,286,534,541)$ & $(72,94,173,197)$ & $(140,161,549,566)$ \\
& $(103,242)$ & $(103,545)$ & $(85,202)$ & $(150,553)$ \\
\hline
\end{tabular}

TABLE I: The lower limits on the masses of the four neutralino states and two chargino states in each model in the form $\left(m_{\chi_{1}^{0}}, m_{\chi_{2}^{0}}, m_{\chi_{3}^{0}}, m_{\chi_{4}^{0}}\right)[\mathrm{GeV}]$ followed by $\left(m_{\chi_{1}^{ \pm}}, m_{\chi_{2}^{ \pm}}\right)[\mathrm{GeV}]$, with the given set of parameters, following from the experimental lower bound on the mass of the lightest chargino. For the mirage mediation model with $\alpha=2$ the limit is not from the chargino mass bound, but from the requirement of the nontachyonic spectrum.

\section{Comparison of the patterns of neutralino and chargino masses}

In Table I we show the lightest neutralino and chargino masses, which satisfy the experimental limit for the mass of the lightest chargino [21] for a particular parameter point. These masses are calculated using SOFTSUSY(v.3.0.13) [46]. The absolute value of the Higgsino mixing parameter is determined by the condition of radiative electroweak symmetry breaking (REWSB), and thus depends on the soft scalar mass parameter $m_{0}$. The parameter $m_{0}$ also enters the radiative corrections through the scalar masses. The masses of the neutralinos in Table $\prod$ have been calculated for $m_{0}=200 \mathrm{GeV}$ and $m_{0}=1 \mathrm{TeV}$ for both the mSUGRA and AMSB models, and $c_{i}=a_{i}=1$ for the mirage mediation. The sign of the $\mu$ parameter was chosen positive. Changing it to the negative value can reduce the neutralino masses by a few GeV's, but this may lead to conflict with the $b \rightarrow s \gamma$ constraint. In mSUGRA the trilinear $A$-parameter was set to zero. Changing that to nonzero values also may decrease the lightest neutralino masses by a few GeV's. These masses demonstrate the effect of the sfermion spectrum on the neutralino and chargino masses for the models that we have studied in this paper. For anomaly mediated supersymmetry breaking, we see the familiar result that the lightest neutralino is closely degenerate with the lightest chargino. The neutralino spectrum in AMSB models is typically heavier than in the case of mSUGRA. In the case of mirage mediation, the mass difference of the lightest and heaviest neutralino masses is smaller as compared to this mass difference in the other models. In addition, especially in the $\alpha=2$ model, the $\mu$-parameter is smaller as compared to its value in the other models, leading to larger mixing of the gaugino and Higgsino components. The much heavier spectrum in the $\alpha=2$ mirage mediation model is due to the tachyonic stops in the spectrum for the lighter particles.

The masses of the neutralinos are plotted in Fig. 1 for the mSUGRA, AMSB and the mirage mediation scenarios, respectively. In mSUGRA the lightest neutralino is more than $80 \%$ bino, while the second lightest one is more than $70 \%$ wino. In AMSB, $\chi_{1}^{0}$ is almost $100 \%$ wino and $\chi_{2}^{0}$ bino. In the mirage mediation pattern with $\alpha=1$, the compositions of the two lightest neutralinos are more evenly divided between bino and wino, but the lightest one is dominantly bino and the second lightest one wino. For small $M_{2}$, also the Higgsino component is nonnegligible in both. For $\alpha=2$, the $\mu$-parameter becomes relatively small, and both $\chi_{1}^{0}$ and $\chi_{2}^{0}$ are more than $90 \%$ Higgsinos. However, for the small values of $M_{2}$, the LSP is not a neutralino as we will discuss later in Sec. VI (see Fig. 12 b).

The ratio of the mass parameters, $\left|M_{3}\right| /\left|M_{2}\right|$ is known to differ drastically in different models. When the radiative corrections are taken into account, the ratios for each pattern discussed above is calculated to be

$$
\left.\frac{\left|M_{3}\right|}{\left|M_{2}\right|}\right|_{\mathrm{mSUGRA}}=3.3,\left.\frac{\left|M_{3}\right|}{\left|M_{2}\right|}\right|_{\mathrm{AMSB}}=7.1,\left.\frac{\left|M_{3}\right|}{\left|M_{2}\right|}\right|_{\alpha=1}=1.4,\left.\frac{\left|M_{3}\right|}{\left|M_{2}\right|}\right|_{\alpha=2}=0.77 \text {. }
$$

If one applies the ratio to the masses of the particles, one finds

$$
\left.\frac{\left|m_{\tilde{g}}\right|}{\left|m_{\chi_{1}^{ \pm}}\right|}\right|_{\mathrm{mSUGRA}}=3.8-4.3,\left.\frac{\left|m_{\tilde{g}}\right|}{\left|m_{\chi_{1}^{ \pm}}\right|}\right|_{\mathrm{AMSB}}=7.3-7.4,\left.\frac{\left|m_{\tilde{g}}\right|}{\left|m_{\chi_{1}^{ \pm}}\right|}\right|_{\alpha=1}=1.7,\left.\frac{\left|m_{\tilde{g}}\right|}{\left|m_{\chi_{1}^{ \pm}}\right|}\right|_{\alpha=2}=0.9,
$$

with other parameter values as given in Table 1. Thus, very large mass ratio of gluino and chargino hints to an AMSB type breaking, while small value hints towards mirage type of supersymmetry breaking. 

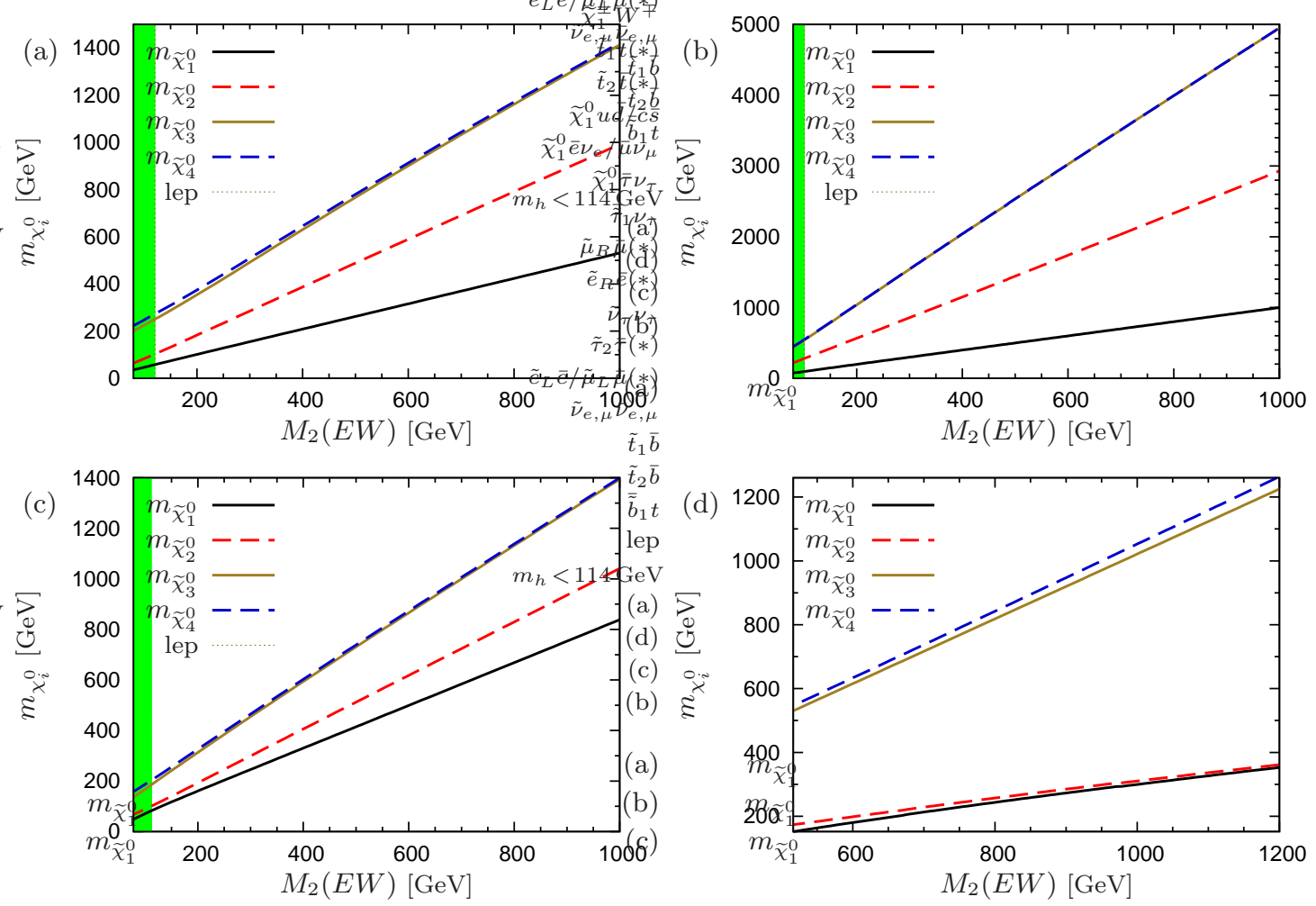

FIG. 1: Masses of the neutralinos in the (a) mSUGRA, (b) AMSB, and for mirage mediation models with (c) $\alpha=1$ and (d) $\alpha=2$. Here $\tan \beta=10, \operatorname{sgn}(\mu)=+1, A_{0}=0, m_{0}=1 \mathrm{TeV}$ (mSUGRA) and $1.5 \mathrm{TeV}$ (AMSB). lep-denoted shading indicates the violation of the LEP sparticle mass limits.

\section{THE GENERAL UPPER BOUND ON THE MASS OF THE LIGHTEST NEUTRALINO}

In this Section we shall consider a general upper bound on the mass of the lightest neutralino that follows from the structure of the neutralino mass matrix. Since some of the neutralino masses resulting from diagonalization of the mass matrix (A5) can be negative, we shall for our purposes consider the squared mass matrix $\hat{\mathcal{M}}^{\dagger} \hat{\mathcal{M}}$. This squared mass matrix can be written as

$$
\mathcal{M}_{0}^{\dagger} \mathcal{M}_{0}=\left(\begin{array}{cccc}
M_{1}^{2}+M_{Z}^{2} s_{w}^{2} & -M_{Z}^{2} c_{w} s_{w} & -M_{Z} s_{w}\left(M_{1} c_{\beta}+\mu s_{\beta}\right) & M_{Z} s_{w}\left(M_{1} s_{\beta}+\mu c_{\beta}\right) \\
-M_{Z}^{2} c_{w} s_{w} & M_{2}^{2}+M_{Z}^{2} c_{w}^{2} & M_{Z} c_{w}\left(M_{2} c_{\beta}+\mu s_{\beta}\right) & -M_{Z} c_{w}\left(M_{2} s_{\beta}+\mu c_{\beta}\right) \\
-M_{Z} s_{w}\left(M_{1} c_{\beta}+\mu s_{\beta}\right) & M_{Z} c_{w}\left(M_{2} c_{\beta}+\mu s_{\beta}\right) & M_{Z}^{2} c_{\beta}^{2}+\mu^{2} & M_{Z}^{2} c_{\beta} s_{\beta} \\
M_{Z} s_{w}\left(M_{1} s_{\beta}+\mu c_{\beta}\right) & -M_{Z} c_{w}\left(M_{2} s_{\beta}+\mu c_{\beta}\right) & -M_{Z}^{2} c_{\beta} s_{\beta} & M_{Z}^{2} s_{\beta}^{2}+\mu^{2}
\end{array}\right),
$$

where $c_{W}=\cos \theta_{W}, s_{W}=\sin \theta_{W}, c_{\beta}=\cos \beta$ and $s_{\beta}=\sin \beta$, respectively. An upper bound on the squared mass of the lightest neutralino $\chi_{1}^{0}$ can be obtained by using the fact that the smallest eigenvalue of $\mathcal{M}_{0}^{\dagger} \mathcal{M}_{0}$ is smaller than the smallest eigenvalue of its upper left $2 \times 2$ sub-matrix

$$
\left(\begin{array}{cc}
M_{1}^{2}+M_{Z}^{2} \sin ^{2} \theta_{W} & -M_{Z}^{2} \sin \theta_{W} \cos \theta_{W} \\
-M_{Z}^{2} \sin \theta_{W} \cos \theta_{W} & M_{2}^{2}+M_{Z}^{2} \cos ^{2} \theta_{W}
\end{array}\right),
$$

thereby resulting in the tree-level upper bound [5]

$$
M_{\tilde{\chi}_{1}^{0}}^{2} \leq \frac{1}{2}\left(M_{1}^{2}+M_{2}^{2}+M_{Z}^{2}-\sqrt{\left(M_{1}^{2}-M_{2}^{2}\right)^{2}+M_{Z}^{4}-2\left(M_{1}^{2}-M_{2}^{2}\right) M_{Z}^{2} \cos 2 \theta_{W}}\right) .
$$




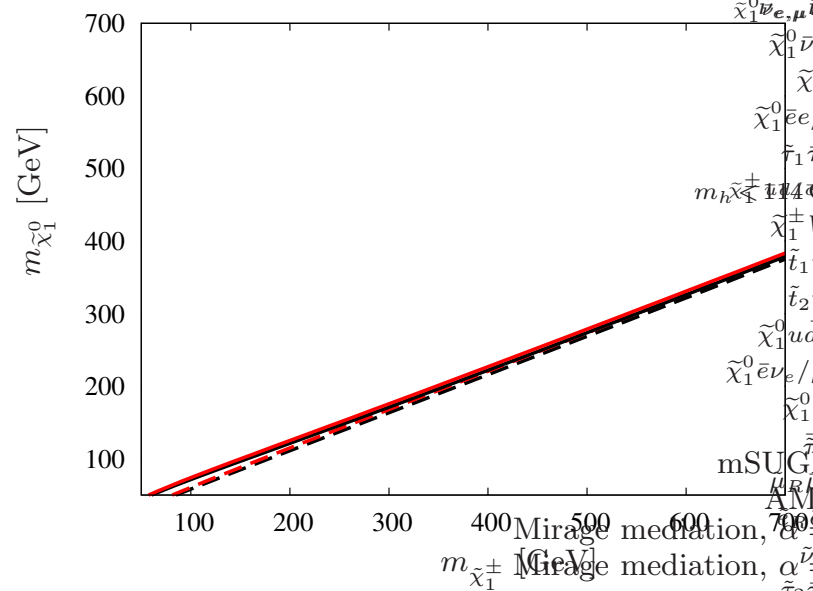

(a)

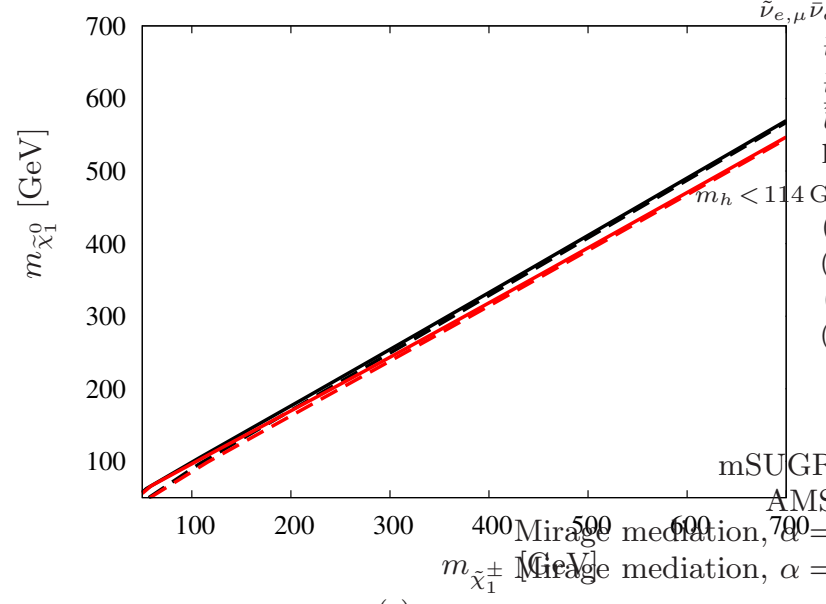

(c)

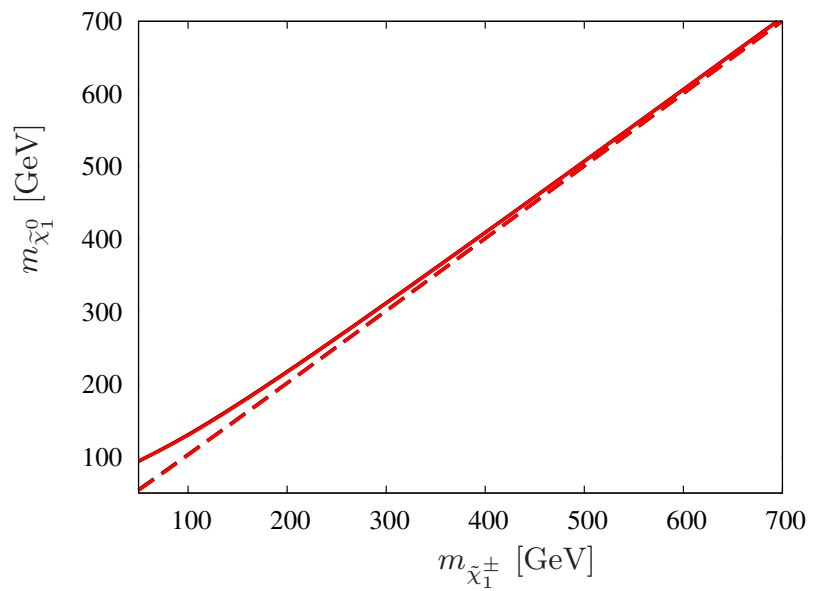

(b)

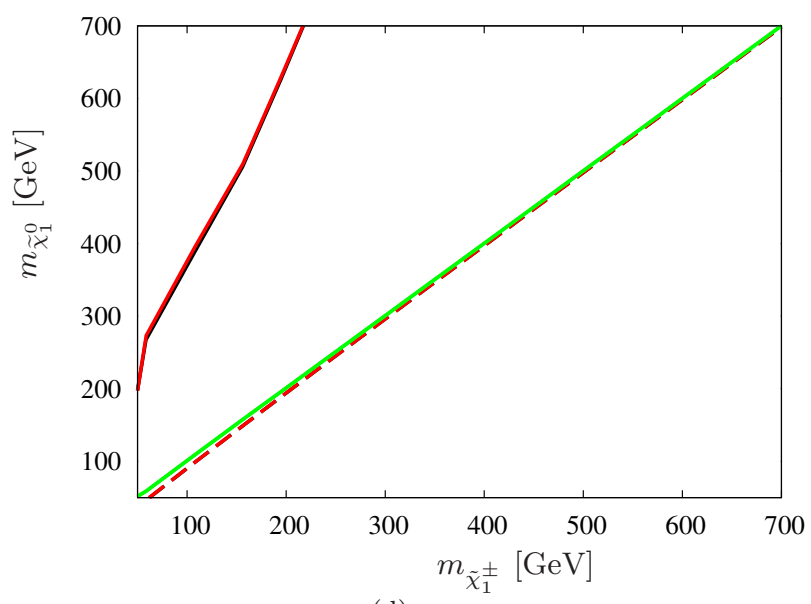

(d)

FIG. 2: Upper limit (solid line) and the mass (dashed line) for the lightest neutralino mass for (a) $\mathrm{mSUGRA}$ with $m_{0}=1$ $\mathrm{TeV}$ and $A=0$, (b) AMSB with $m_{0}=1 \mathrm{TeV}$, and mirage mediation with $a=c=1$ and with (c) $\alpha=1$, and (d) with $\alpha=2$, calculated at tree-level (red/gray) and with radiative corrections added (black) as a function of the mass of the lighter chargino. In all the plots $\tan \beta=10$ and $\operatorname{sign}(\mu)=+1$.

We emphasize that the upper bound (3.3) is independent of the supersymmetry conserving parameter $\mu$ and also independent of $\tan \beta$, but depends on the supersymmetry breaking gaugino mass parameters $M_{1}$ and $M_{2}$. Despite this dependence on the unknown supersymmetry breaking parameters Eq. (3.3) leads to a useful bound on $M_{\chi_{1}^{0}}$. An alternative bound on the mass of the lightest neutralino can be obtained by considering the bottom-right $2 \times 2$ sub-matrix

$$
\left(\begin{array}{cc}
M_{Z}^{2} \cos ^{2} \beta+\mu^{2} & -M_{Z}^{2} \cos \beta \sin \beta \\
-M_{Z}^{2} \cos \beta \sin \beta & M_{Z}^{2} \sin ^{2} \beta+\mu^{2}
\end{array}\right),
$$

leading to an upper bound

$$
M_{\chi_{1}^{0}}^{2} \leq|\mu|^{2}
$$

This bound, when supplemented by the electroweak symmetry breaking condition

$$
\frac{1}{2} M_{Z}^{2}=\frac{\left(m_{1}^{2}-m_{2}^{2} \tan ^{2} \beta\right)}{\left(\tan ^{2} \beta-1\right)}-|\mu|^{2}
$$




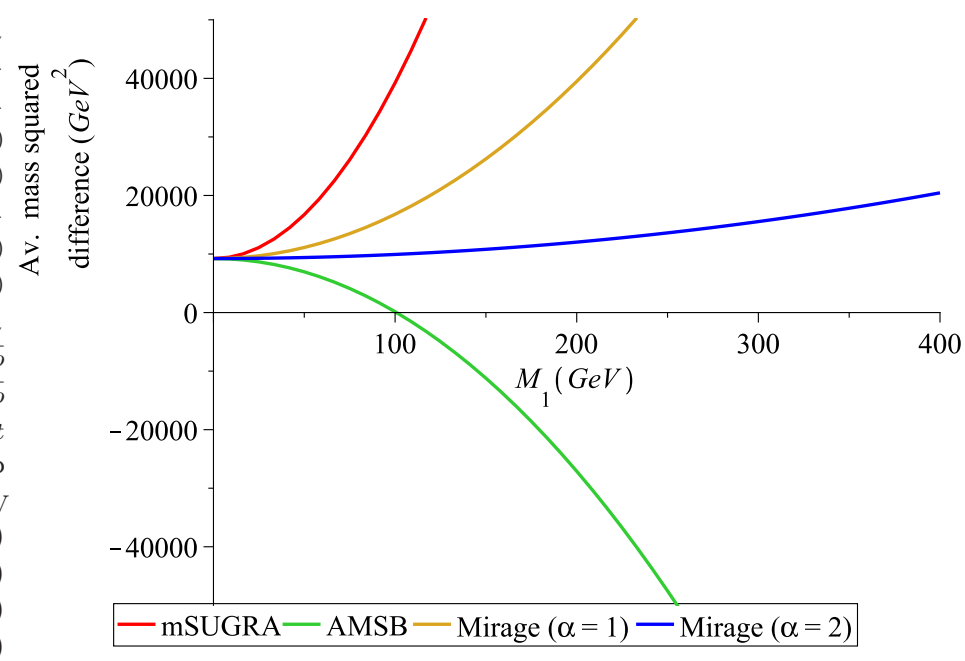

FIG. 3: The sum rule (4.1) plotted for different gaugino mass patterns.

leads to the upper bound

$$
M_{\chi_{1}^{0}} \leq|\mu|=\left[\frac{\left(m_{1}^{2}-m_{2}^{2} \tan ^{2} \beta\right)}{\left(\tan ^{2} \beta-1\right)}-\frac{1}{2} M_{Z}^{2}\right]^{\frac{1}{2}}
$$

The importance of this bound stems from the fact that it relates the two sectors, namely the supersymmetry breaking gaugino masses and the Higgs masses. One can then use the RG evolution for the parameters on the RHS of the bounds to evaluate the bound. Note that the RG equations for the parameters on the RHS of the bounds involve the gaugino masses, and will, therefore, depend on the boundary conditions for the gaugino masses, and hence on the different supersymmetry breaking models for the gaugino mass parameters. In Fig. 2 we have plotted the upper limits for the lightest neutralino mass following from (3.3) for the different supersymmetry breaking models as a function of $m_{\tilde{\chi}_{1}^{ \pm}}$. For all the four models studied we plot the tree-level masses, and the two-loop radiatively corrected masses calculated using SOFTSUSY(v.3.0.13) [46]. In Fig. 22 d, we plot also the upper limit from Eq. (3.7), which gives the lowest mass upper bound in this case. Note that the mass of the lighter of the charginos is close to the value of the $\mu$-parameter. The neutralino masses have been calculated assuming $\tan \beta=10$, and the other parameters as indicated in the Figure. We note that all gaugino masses can receive radiative corrections up to $20 \%$, and, thus, difference between tree level and radiatively corrected neutralino and chargino masses can be significant in all models studied in this paper, although the difference is not explicit in Fig. 2 due to similar magnitude of correction for both the neutralino and the chargino.

\section{SUM RULES}

We recall that in the minimal AMSB model, the mass difference between the lightest chargino and the lightest neutralino is small. The close proximity of the lightest neutralino and chargino masses is a direct consequence of Eq. (2.15), which gives for the ratios of the gaugino mass parameters $\left|M_{1}\right|:\left|M_{2}\right|:\left|M_{3}\right| \simeq 2.8: 1: 7.1$, after taking into account the next to leading order radiative corrections and the weak scale threshold corrections [31] as in (2.19). Thus, the winos are the lightest neutralinos and charginos, and one would expect that the lightest chargino is only slightly heavier than the lightest neutralino in all anomaly mediated models. It is not feasible to obtain sum rules for the masses of the neutralino states, since the physical neutralino mass matrix is a $4 \times 4$ matrix. However, from the trace of the squares of the neutralino and chargino mass matrices, one obtains a sum rule, which does not contain the Higgs mixing parameter $\mu$, but which is present in the mass matrices. The sum rule can be written as

$$
2 \sum_{i=1}^{2} M_{\tilde{\chi}_{i}^{ \pm}}^{2}-\sum_{i=1}^{4} M_{\tilde{\chi}_{i}^{0}}^{2}=\left[M_{2}^{2}-M_{1}^{2}\right]+4 M_{W}^{2}-2 M_{Z}^{2}
$$

By using the gaugino mass pattern for a specific model the sum rule (4.1) can be expressed as as function of any of the gaugino masses. This is shown in Fig. 3. The average mass difference in the AMSB is first positive, but then 
quickly turns negative, while in the minimal SUGRA model it is always positive. In the mirage mediation model the behavior is determined by the parameter $\alpha$ with a low value leading to a mSUGRA-like curve. Increasing $\alpha$ decreases the gradient of the curve until $\alpha=2.17$ (the value for which $M_{1}=M_{2}$ ) leads to a constant positive value. We note that this sum rule could be used as a signature for different supersymmetry breaking models, and in the case of mirage mediation it might be useful for determining the value of $\alpha$, which can be calculated from the sum rule for specific values of gaugino masses.

\section{DECAYS OF NEUTRALINOS AND CHARGINOS}

In this Section we discuss the decays of charginos and neutralinos in different supersymmetry breaking models that we have discussed in this paper. As noted earlier, charginos and neutralinos are mass eigenstates, which are model-dependent linear combinations of charged or neutral gauginos and Higgsinos. Since the mass matrices of charginos and neutralinos depend on parameters $M_{1}$ and $M_{2}$, which are model dependent, the decays will depend on the model under consideration. As such the decay patterns of charginos and neutralinos can serve as a window on the underlying supersymmetry breaking mechanism in the gaugino sector. Here we shall mostly focus on two-body tree-level decays of neutralinos and charginos, if they are kinematically possible. If the neutralino or the chargino is sufficiently heavy, then two-body decays into a $W, Z^{0}$, or a Higgs boson and a lighter neutralino or chargino are the dominant decay modes. Since in supersymmetric models with minimal particle content, the lightest Higgs boson is relatively light, the two-body decay containing the light Higgs boson is expected to be the dominant decay mode over a large region of parameter space. However, if some squarks or sleptons are relatively light, the two body treelevel decays of a heavy neutralino or chargino to quark-squark or lepton-slepton may be important. However, these decays are phenomenologically less important at a hadron collider like LHC, where neutralinos and charginos would be produced from the decays of strongly interacting squarks and gluinos. Neutralinos and charginos, which are heavier than squarks, would be hard to study at a hadron collider.

We recall that if a heavier $\tilde{\chi}_{i}^{0}(i=2,3,4)$ or a chargino $\tilde{\chi}_{j}^{+}(j=1,2)$ is produced at a collider, it will decay via a cascade until the lightest neutralino $\left(\tilde{\chi}_{1}^{0}\right)$ is produced. Thus, we are here interested in the branching ratios for the two-body decays

$$
\begin{array}{llll}
\tilde{\chi}_{i}^{0} \rightarrow \tilde{\chi}_{j}^{0}+Z^{0}, & \tilde{\chi}_{i}^{0} \rightarrow \tilde{\chi}_{j}^{ \pm}+W^{\mp}, & \tilde{\chi}_{i}^{+} \rightarrow \tilde{\chi}_{j}^{0}+W^{+}, & \tilde{\chi}_{i}^{+} \rightarrow \tilde{\chi}_{j}^{+}+Z^{0}, \\
\tilde{\chi}_{i}^{0} \rightarrow \tilde{\chi}_{j}^{0}+H_{k}^{0}, & \tilde{\chi}_{i}^{0} \rightarrow \tilde{\chi}_{j}^{ \pm}+H^{\mp}, & \tilde{\chi}_{i}^{+} \rightarrow \tilde{\chi}_{j}^{0}+H^{+}, & \tilde{\chi}_{i}^{+} \rightarrow \tilde{\chi}_{j}^{+}+H_{k}^{0} .
\end{array}
$$

These two body decays will dominate any tree-level three-body decays mediated by virtual squark or slepton exchange. These decays will also dominate any two-body decay, which is forbidden at the tree level, but which can proceed via loops, such as $\tilde{\chi}_{i}^{0} \rightarrow \tilde{\chi}_{j}^{0}+\gamma$.

If some of the neutralinos and charginos are heavier than some of the squarks and sleptons, then the two-body decays

$$
\tilde{\chi} \rightarrow q \tilde{q}, l \tilde{l}
$$

can compete with the two-body decays into $W, Z^{0}, H$ discussed above. The analytical expressions for the branching ratios of charginos and neutralinos into $W, Z^{0}$, and Higgs bosons, as well as into squarks/sleptons for arbitrary neutralino and chargino mixing angles are given in [47].

For the evaluation of branching ratios of charginos and neutralinos, we have calculated the spectra of the supersymmetric particles using SOFTSUSY(v.3.0.13) [46], and the decays of the supersymmetric particles using SUSYHIT(v.1.3 with SDECAY v1.3b/HDECAY v3.4) [48]. In the following analysis we have used the parameter values $\tan \beta=10$ and $\operatorname{sign}(\mu)=+1$ for all models. In addition, for the mSUGRA and AMSB models, we have used $m_{0}=1$ TeV. For the mirage mediation of supersymmetry breaking, we have used $\alpha=1 / 2$ and $\alpha=1$ with $c_{i}=a_{i}=1$. The mirage mediation scenarios with higher value for $\alpha$ can lead to squark LSP or tachyons, as will be discussed in next Section. While calculating the decay rates of charginos and neutralinos, we have imposed the experimental constraints following from LEP sparticle mass limits and the LEP lower bound on the lightest Higgs boson mass. Thus, in each of the following figures, the green (dark in grayscale) color denotes the area where LEP sparticle mass limits are violated, and light blue color (light) denotes the area where the lightest Higgs mass is below $114 \mathrm{GeV}$. In addition, the vertical Isp-denoted line in the mirage $\alpha=1$ figures indicates the minimum mass for which the neutralino is the LSP. The bsg-denoted line represents the $B R(b \rightarrow s \gamma)$ constraint. In the anomaly mediated supersymmetry breaking models, the bsg line practically coincides with the Higgs mass limit for these parameters, and is not drawn explicitly in order to reduce too many curves in the figure. (In the mSUGRA figures the constraint is obeyed throughout the mass ranges.) The LEP and $b \rightarrow s \gamma$ constraints are calculated with micrOmegas (see Sec. VI). An asterisk after 

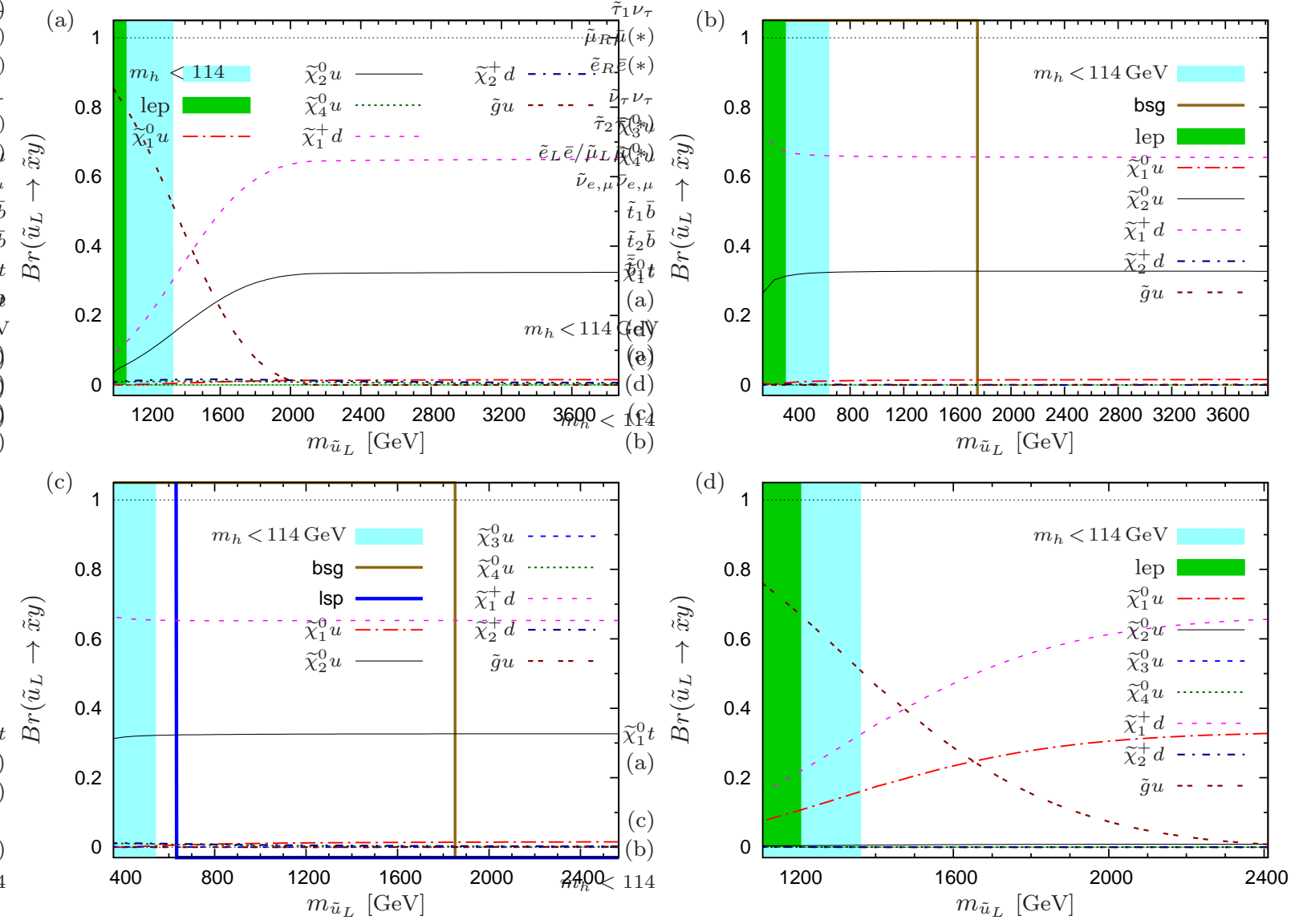

FIG. 4: Branching ratios for the two body decays of $\tilde{u}_{L}$ to gauginos and Higgsinos in (a) mSUGRA model, (b) mirage mediation scenario with $\alpha=0.5$ and (c) $\alpha=1$, and (d) the AMSB scenario. Shadings specify the region where the LEP mass limits are not satisfied (dark) or the lightest Higgs mass is below $114 \mathrm{GeV}$ (light). See the text for more details.

the decay mode indicates that the charge conjugated mode is also included in the plotted value. A label of the form $\widetilde{\chi}_{1}^{0} \bar{x} x / \bar{y} y$ indicates that the plotted value includes both channels $\widetilde{\chi}_{1}^{0} \bar{x} x$ and $\widetilde{\chi}_{1}^{0} \bar{y} y$, and that each channel contributes equally.

We begin by commenting the production of charginos and neutralinos at the LHC. The direct pair production of these particles is rare [49] compared to the pair production of strongly interacting particles [50]. However, neutralinos and charginos are also produced in the cascade decays of squarks and gluinos. As an example, we study the decays of squarks to neutralinos and charginos.

The decay branching ratio of the left-handed u-squark is depicted in Fig. 4. It is seen that if gluino remains lighter than the squark, the decay is dominantly via the strong coupling to gluino and quark, and then further gluino would decay to the lightest neutralino with a quark pair or a gluon. If the two-body decay to quark and gluino is kinematically not possible, the squark decays mainly to the lighter chargino and quark, $\tilde{\chi}_{1}^{+} d$, or to the second lightest neutralino and quark, $\tilde{\chi}_{2}^{0} u$, in mSUGRA and mirage patterns. In the AMSB, the branching ratio to $\tilde{\chi}_{1}^{0} u$ is larger than to $\tilde{\chi}_{2}^{0} u$ This is due to the fact that in the mSUGRA and mirage patterns the neutralino $\chi_{2}^{0}$ is mainly wino, while in the AMSB pattern $\chi_{1}^{0}$ is dominantly wino. The right-handed squark $\tilde{u}_{R}$ decays most of the time to $\chi_{1}^{0} u$ in the mSUGRA and mirage patterns, and to $\chi_{2}^{0} u$ in the AMSB case, since for $\tilde{u}_{R}$ the decay to the bino dominated neutralino is favoured.

For the chosen set of parameters with $m_{0}=1 \mathrm{TeV}$, the squarks are relatively heavy, especially when the experimentally allowed range for $b \rightarrow s \gamma$ is taken into account. Thus one might want to consider the third generation stops, which are lighter than the other squarks due to mixing. In Fig. 5 we have plotted the two-body decay modes of $\tilde{t}_{1}$ to neutralinos, charginos and gluinos as a function of the stop mass. One finds that especially in the case of mSUGRA and AMSB, the cascades produce heavier neutralinos and charginos, e.g. in the AMSB pattern more than $80 \%$ of the lightest stop decays to $\tilde{\chi}_{2}^{0} t$ or $\tilde{\chi}_{1}^{+} b$. This is different from the two mirage mediation scenarios, where the lightness of stop causes it to decay directly to top and the neutralino LSP, or to b-quark, W-boson and neutralino through a 

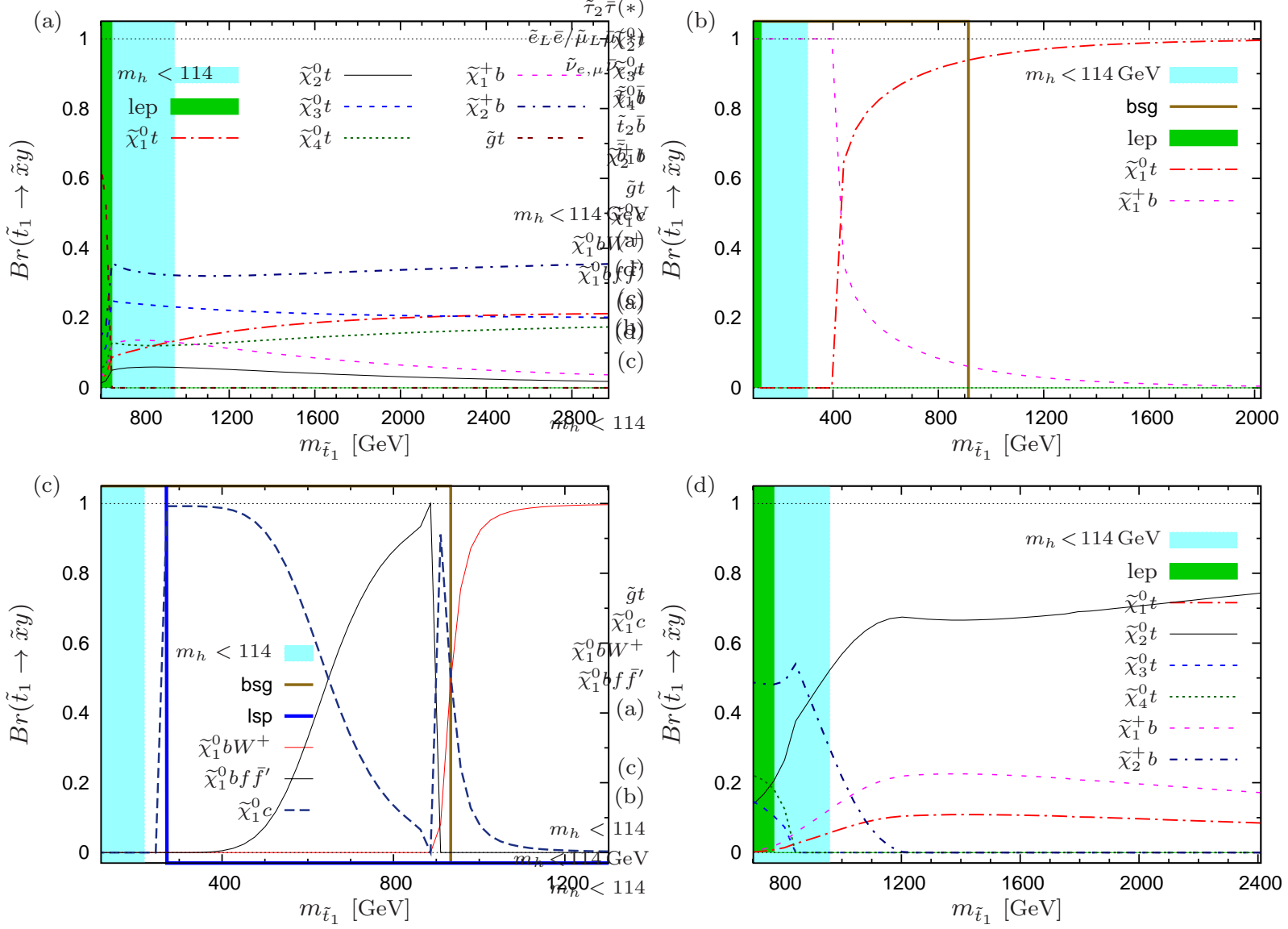

FIG. 5: Branching ratios for the largest decay modes of $\tilde{t}_{1}$ to gauginos and Higgsinos in (a) mSUGRA model, (b) mirage mediation scenario with $\alpha=0.5$ and (c) $\alpha=1$, and (d) the AMSB scenario. Shadings specify the region where the LEP mass limits are not satisfied (dark) or the lightest Higgs mass is below $114 \mathrm{GeV}$ (light). See the text for more details.

\section{3-body process.}

In Fig. [6 we have plotted the dominant decay modes of $\widetilde{\chi}_{2}^{0}$ as a function of its mass for mSUGRA, the mirage mediation models, and the AMSB, respectively. We note from Fig. 6 that the dominant mode of $\widetilde{\chi}_{2}^{0}$ in mSUGRA is into the $\widetilde{\chi}_{1}^{0}$ and the lightest Higgs boson, when kinematically possible, and into $\widetilde{\chi}_{1}^{0}$ and the $Z^{0}$ boson for a light $\widetilde{\chi}_{2}^{0}$. The same applies for the two mirage mediation scenarios; one can see the opening of the Higgs mode to be pushed gradually to higher neutralino masses as the mirage scenario further deviates from the pure mSUGRA by increasing $\alpha$. With an increasing $\alpha$, stop becomes lighter and can be the lightest supersymmetric particle. In the heavier end of the spectrum the lightness of stops enables the stop decay mode. In the AMSB models, where the light chargino and neutralino are almost degenerate in mass, the decay mode to the lighter chargino and W-boson dominates the decay to $\widetilde{\chi}_{1}^{0}$ and the lightest Higgs boson, until the sfermion decay modes become kinematically possible and begin to dominate.

In Fig. 7 we have plotted the dominant two body decay modes of the $\widetilde{\chi}_{3}^{0}$ as a function of its mass for mSUGRA, the mirage mediation models, and the AMSB, respectively. For $\widetilde{\chi}_{3}^{0}$ the chargino- $W$ mode is available in mSUGRA, and it dominates. However, the $\widetilde{\chi}_{2}^{0} Z^{0}$ mode starts competing with increasing mass of $\widetilde{\chi}_{3}^{0}$. In the mirage mediation models the stop-top channel is available in addition to the $\widetilde{\chi}_{1}^{ \pm} W$ and the $Z^{0}$ decay modes. Furthermore, in the mirage mediation scenario, the light stop-top channel takes the role of the dominant decay mode for a large mass range, where the two mirage scenarios give quite similar branching ratios for the $\widetilde{\chi}_{3}^{0}$ decay. This is in contrast to the AMSB scenario, where the $\widetilde{\chi}_{1}^{ \pm} W$ and $\widetilde{\chi}_{1}^{0} Z^{0}$ modes dominate.

In Fig. 8 we have plotted the dominant two body decay modes of the $\widetilde{\chi}_{4}^{0}$ as a function of mass of $\widetilde{\chi}_{4}^{0}$. In mSUGRA and AMSB scenarios the $\widetilde{\chi}_{1}^{ \pm} W$ mode dominates, whereas in the mirage mediation the stop-top channel has the largest branching fraction. For the relevant mass range, in the two mirage scenarios, the $\widetilde{\chi}_{4}^{0}$ decays look similar. Furthermore, the light Higgs decay modes are present in each breaking scenario.

In Fig. 9 the dominant decay modes of $\widetilde{\chi}_{1}^{ \pm}$are plotted as a function of its mass for the different supersymmetry 

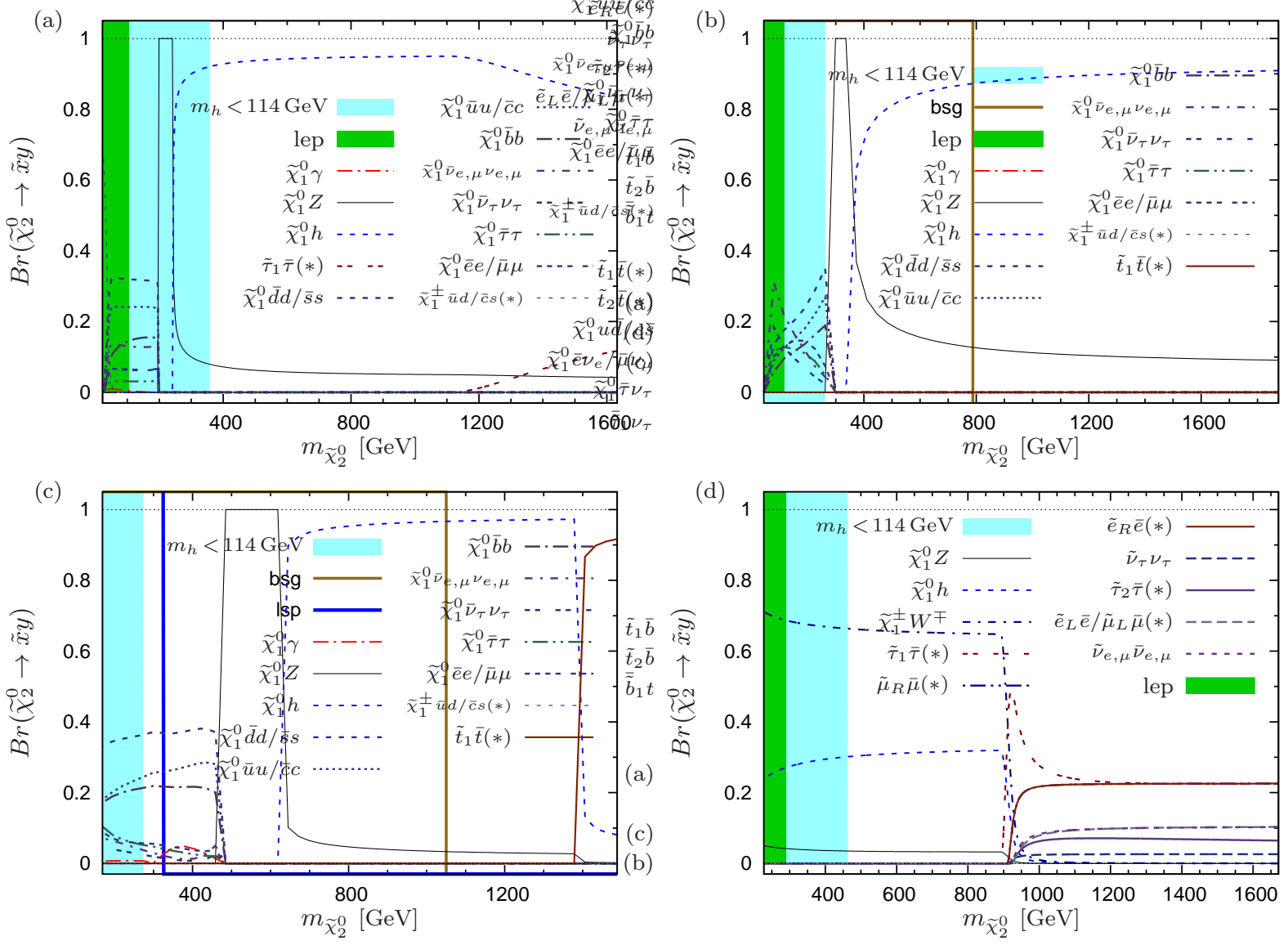

FIG. 6: Branching ratios for the two body decays of $\tilde{\chi}_{2}^{0}$ in (a) mSUGRA model, (b) mirage mediation scenario with $\alpha=0.5$ and (c) $\alpha=1$, and (d) the AMSB scenario. Also the three body channels are shown where no two body decays are possible. The $(*)$ in the channel label signifies that the channel includes also the charge conjugated final state. Shadings specify the region where the LEP mass limits are not satisfied (dark) or the lightest Higgs mass is below $114 \mathrm{GeV}$ (light).

breaking models. In mSUGRA the only possible two-body decay mode, until the slepton (stau) channel becomes available, is the $\widetilde{\chi}_{1}^{0} W$ mode. In the mirage mediation scenario the lightness of the stop enables the $\tilde{t}_{1} \bar{b}$ mode, which becomes the dominant one for $\alpha=1$. The other available channel is the $\widetilde{\chi}_{1}^{0} W$ mode, which in fact is the only two-body mode in the $\alpha=0.5$ case, thus making the two mirage scenarios distinguishable with respect to the lighter chargino decay. Also, the neutralino LSP and the $b \rightarrow s \gamma$ requirements push the chargino mass to be reasonably heavy in the $\alpha=1$ mirage scenario. In the AMSB models the lighter chargino mass is so close to the lightest neutralino mass that only three body decays are available.

Finally, in Fig. 10 we plot the dominant two body decay modes of the $\widetilde{\chi}_{2}^{ \pm}$as a function of its mass for the models discussed in this paper. In mSUGRA the $W$ and $Z^{0}$ modes dominate for the low mass range, but eventually the light Higgs channel becomes a competing mode. In the AMSB scenario the light Higgs, $Z^{0}$ and $W$ modes have approximately the same branching ratio. In contrast to the other models, in the mirage mediation the $\tilde{t}_{1} \bar{b}$ mode dominates by a large margin.

In general, it is obvious that in the AMSB models the close proximity of the lightest neutralino and the lighter chargino is important in the decays of heavier neutralinos and chargino as well. In addition to forcing the lighter chargino to decay via a three body mode, this feature makes the second lightest neutralino to decay dominantly into the lighter chargino and $W$ boson or other charged sparticles. The predictions of the anomaly mediated supersymmetry breaking and the mSUGRA are very different here.

In mirage mediation the mass spectrum for neutralinos and charginos is more tightly packed, and thus for $\chi_{2}^{0}$ and $\chi_{1}^{ \pm}$ it is expected that they decay via a three body mode, if the mass scale is light enough, which again is a distinguishing feature as compared to the mSUGRA and AMSB models. The lightness of stop allows in many cases the stop decay channel in the mirage mediation models, which then becomes the dominant decay mode. 

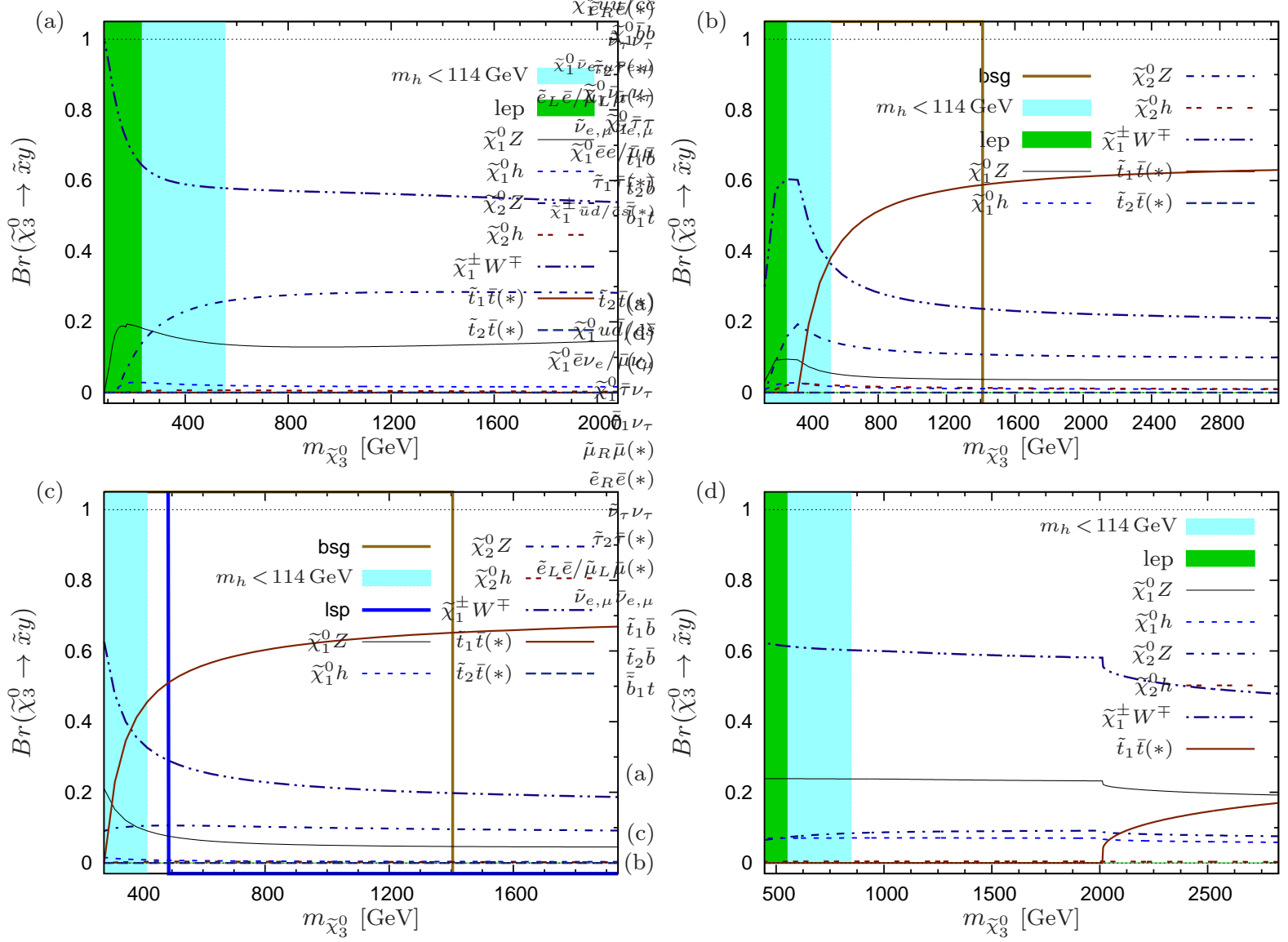

FIG. 7: Branching ratios for the decays of $\widetilde{\chi}_{3}^{0}$ in (a) mSUGRA model, (b) mirage mediation scenario with $\alpha=0.5$ (c) in the mirage mediation scenario with $\alpha=1$, and (d) the AMSB scenario.

\section{RELIC DENSITY}

In this Section, we study the implications of different patterns of supersymmetry breaking gaugino masses for the relic density of lightest neutralino, and the constraints imposed on the parameter space by the precise limits on the relic density obtained by the Wilkinson Microwave Anisotropy Probe (WMAP) satellite. Requiring the lightest neutralino to form all of the dark matter as a thermal relic is very limiting constraint on the parameter space, and it should be kept in mind that the possible dark matter might also be created non-thermally or the excess thermal production diluted for example by an entropy increase after the freeze-out [51 53]. Therefore we refer to the WMAP constrained parameter space as a WMAP-preferred relic density area.

The relic density in the mSUGRA [54 60] and AMSB [31, 61 64] scenarios has been studied extensively. Neutralino dark matter in the mirage mediation SUSY breaking model has been considered in [41, 65 67]. Here we consider the combined information from the relic density and decay modes of the second lightest neutralino. In Fig. 11 the main decay modes of $\widetilde{\chi}_{2}^{0}$ and the WMAP-preferred relic density stripe in the $\left(M_{\widetilde{\chi}_{1}^{ \pm}}, \tan \beta\right)$ plane are plotted for mSUGRA $\left(A_{0}=0, m_{0}=120 \mathrm{GeV}\right.$ and $\left.m_{0}=1 \mathrm{TeV}\right)$, AMSB $\left(m_{0}=5 \mathrm{TeV}\right)$, and mirage mediation $\left(\alpha=1\right.$ with $\left.a_{i}=c_{i}=1\right)$ for $\operatorname{sign}(\mu)=+1$. The spectrum is calculated using SOFTSUSY (v.3.0.13) [46], and the relic density and constraints using micrOmegas (v.2.2.CPC.i) [68]. For the relic density, we use the WMAP combined three year limits [18]

$$
\Omega_{C D M} h^{2}=0.11054_{-0.00956}^{+0.00976} \quad(2 \sigma)
$$

In the figures below, the filling denoted by wmap is the WMAP preferred region. For the $b \rightarrow s \gamma$ experimental branching fraction, the two sigma world average has been used [69], $B R(b \rightarrow s \gamma)=\left(355 \pm 24_{-10}^{+9} \pm 3\right) \times 10^{-6}$, and for the particle masses the limits of [68] are applied. In the figures, lep shows an area where the experimental sparticle mass limits are not met, rge shows an area where there are tachyons or no radiative EWSB, and Isp the area where neutralino is not the LSP. The curve $m_{h}=114 \mathrm{GeV}$ is depicted in the figures for the reference (dash-dotted line denoted by $\mathrm{h}$ ). 

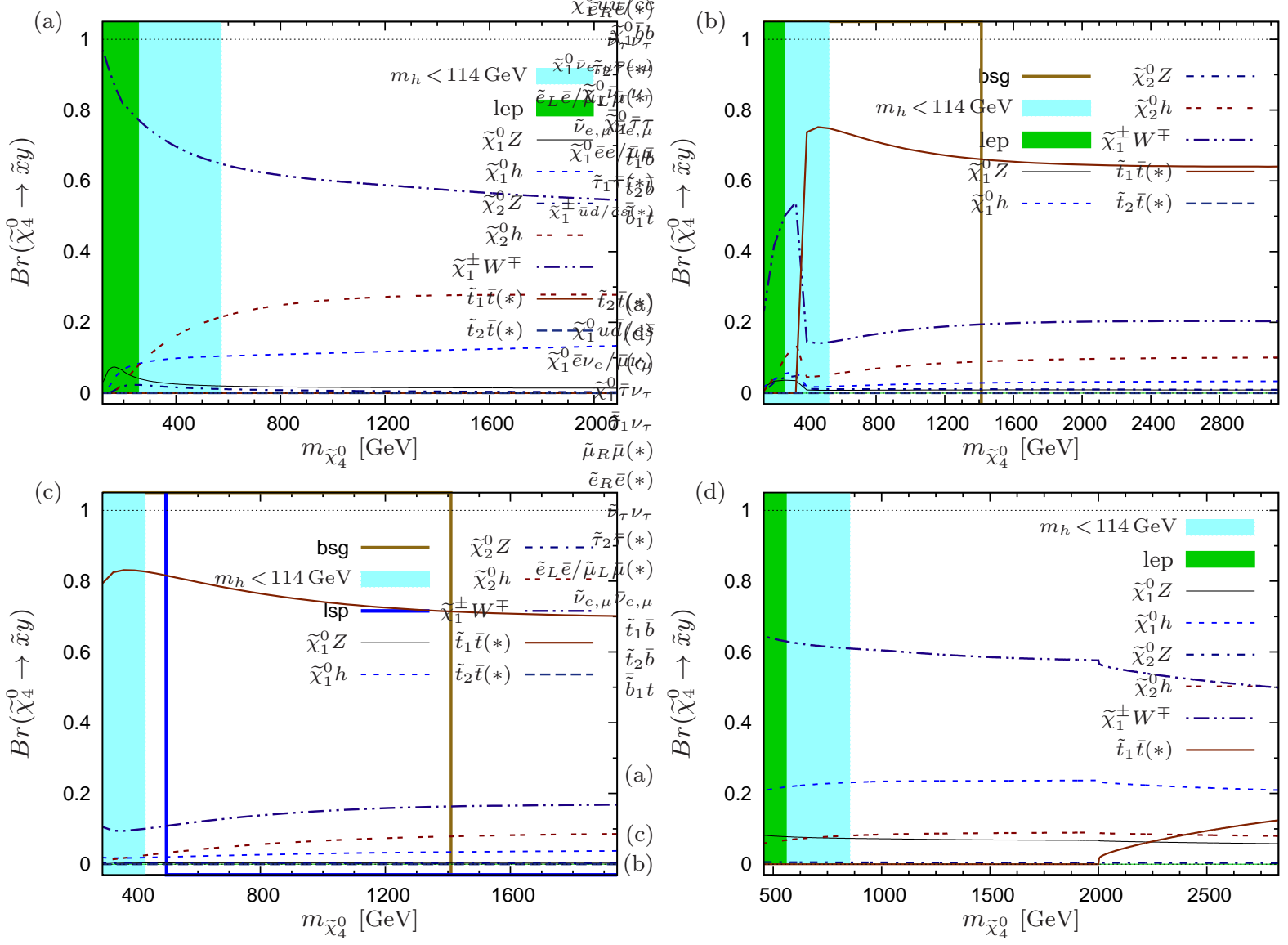

FIG. 8: Branching ratios for the decays of $\tilde{\chi}_{4}^{0}$ in (a) mSUGRA model, (b) mirage mediation scenario with $\alpha=0.5$ and (c) $\alpha=1$, and (d) the AMSB scenario.

In the mSUGRA scenario the WMAP preferred relic density area can be obtained either for a small $m_{0}$ with moderate $\tan \beta$, where the scalars are light and stau coannihilates with light neutralino, or for a larger $m_{0}$ with quite large $\tan \beta$, where $\widetilde{\chi}_{1}^{0}$ annihilates through Higgs channel. The lightest neutralino is very bino-like, which in general results in a large relic density. In Fig. 117 the WMAP preferred region is shown for $m_{0}=120 \mathrm{GeV}$. This is achieved via coannihilation with stau for $m_{\tilde{\chi}_{1}^{ \pm}}<500 \mathrm{GeV}$ and $\tan \beta<20$. The dominant decay mode along the WMAP stripe is to $\tilde{\tau}_{1} \tau$-pair until various other leptonic modes and the light Higgs mode take over with increasing $\tilde{\chi}_{1}^{ \pm}$mass. While the stau coannihilation region exists also for the heavier bino masses, the WMAP preferred region becomes very narrow due to the relic density mass dependence on the LSP mass. Increasing $m_{0}$ to $200 \mathrm{GeV}$ would raise the WMAP preferred region to around $m_{\tilde{\chi}_{1}^{ \pm}} \sim 500-700 \mathrm{GeV}$ and $12<\tan \beta<25$, but the stripe would be clearly narrower due to stau coannihilation with heavier bino. For $m_{0}=1 \mathrm{TeV}, A_{0}=0$, and $\mu>0$, the dark matter area can be found around $\tan \beta \simeq 50$ [70], as shown in Fig. 11] b. Here both neutralino annihilation through a heavy Higgs resonance and stau coannihilation are effective. Along the WMAP stripe $\widetilde{\chi}_{1}^{0} h$ is the dominant decay mode until $\tilde{\tau}_{1} \tau$ becomes kinematically accessible.

We note that since in the AMSB scenario the lightest neutralino is almost a pure wino for a large region of the parameter space, the relic density for moderate $m_{0}$ values tends to be smaller than the WMAP observation. Therefore a spectrum with rather large LSP mass is required for the AMSB to provide the observed relic density. In Fig. 11: the value of $m_{0}=5 \mathrm{TeV}$ has been used. The WMAP region occurs for $m_{\chi_{1}^{ \pm}} \simeq 2 \mathrm{TeV}$, where the decay modes to slepton-lepton pair dominate the $\widetilde{\chi}_{2}^{0}$-decays.

In mirage mediation scenario (Fig. [11]) with $\alpha=1$ with the mentioned choice of parameters the lightest neutralino is mostly bino-like. Therefore the existence of the WMAP-preferred relic density region in general relies on the coannihilation with the NSLP, which for the large part of the parameter space is stop. (For high $\tan \beta$ the NLSP near the Isp-denoted region is stau.) Taking into account the $b \rightarrow s \gamma$ constraint and requiring that the relic density agrees with the WMAP observations practically removes the WMAP-preferred region. If a ten percent error in the theoretical calculation of the $b \rightarrow s \gamma$ constraint is assumed, the WMAP-stripe on the right of the bsg th.-denoted line 

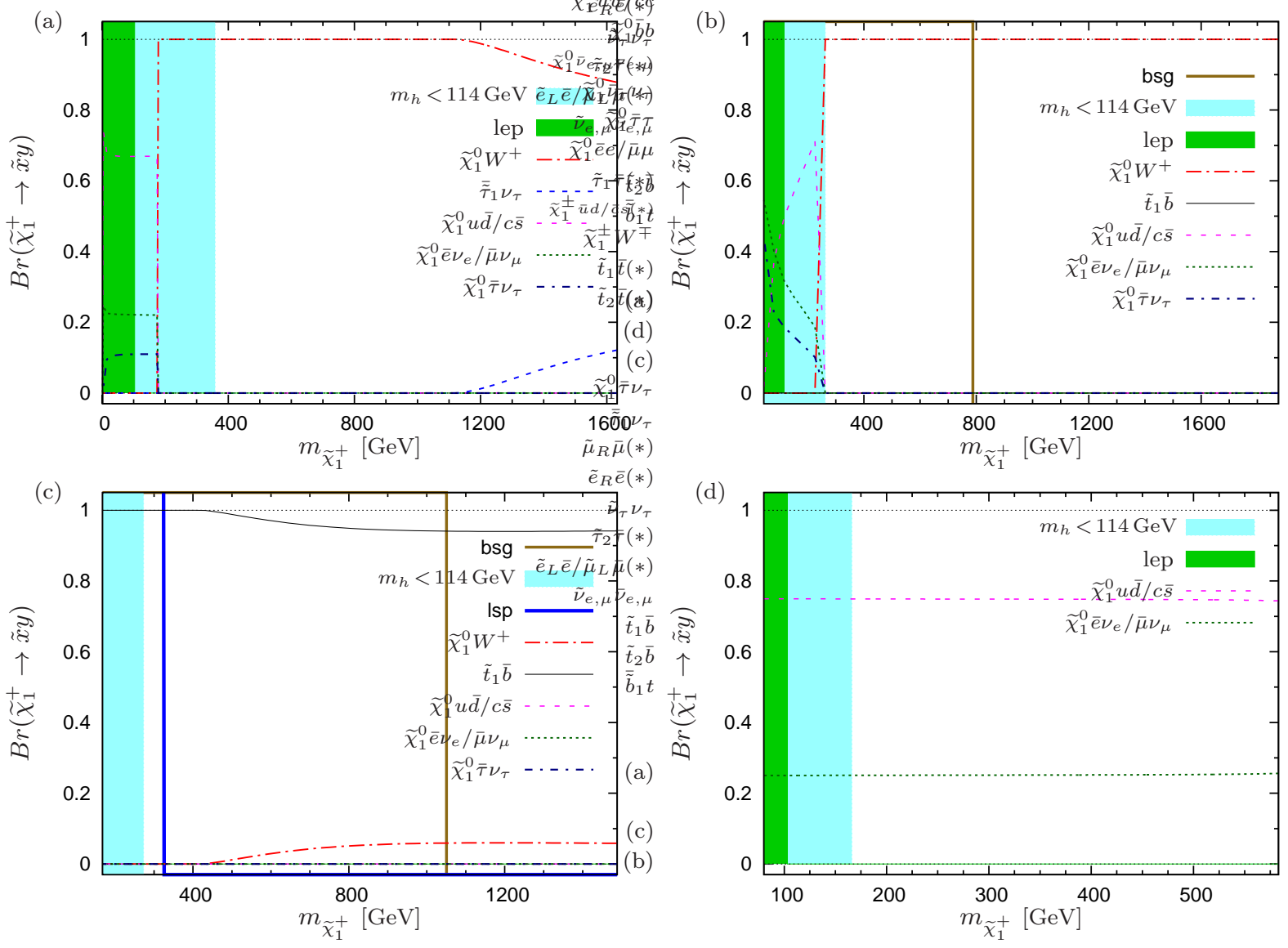

FIG. 9: Branching ratios for the decays of $\tilde{\chi}_{1}^{+}$in (a) mSUGRA model, (b) mirage mediation scenario with $\alpha=0.5$ and (c) $\alpha=1$, and (d) the AMSB scenario.

becomes allowed. The peaking behavior in the relic density is due to the efficient s-channel annihilation through heavy Higgs bosons. Around $\tan \beta=10$ the annihilation rate is enhanced via stops decaying through the Higgs resonance, while around $\tan \beta=18$ it is the lightest neutralino which annihilates directly though the resonance. For a large region of the parameter space the NLSP is the lighter stop, so the WMAP-preferred relic density parameter space follows the neutralino co-annihilation region with stop. The figure is divided into areas where the dominant $\widetilde{\chi}_{2}^{0}$ decay mode exceeds $50 \%$. If the constraints are obeyed, the dominant decay mode for $\widetilde{\chi}_{2}^{0}$ is to $\chi_{1}^{0} h$ or possibly to $\tilde{t}_{1} \bar{t}(*)$ for the WMAP-preferred relic density area.

In Fig. 12a the composition of the lightest neutralino and the WMAP-preferred relic density stripe in the mirage mediation scenario in the $\left(M_{3 / 2}, \alpha\right)$ plane are plotted (for $\tan \beta=10$ and $\operatorname{sign}(\mu)=+1$ ) with $a_{i}=c_{i}=1$. The lightest neutralino composition is painted in RGB-color encoding (i.e., colors (or hues of gray) indicate the particle as shown in the figure color bar; therefore the mixture of the colors (or the shading in black and white) describes the nature of the $\widetilde{\chi}_{1}^{0}$-composition). As the $\alpha$-parameter increases, the lightest neutralino composition makes a transition from the bino-like to very Higgsino-like. Above the Isp-denoted line the stop is the LSP, and therefore that area is not suitable for the dark matter considerations. However, at sufficiently high alpha value the REWSB forces the $\mu$-parameter to very light value, hence reducing the Higgsino like neutralino mass below the sparticle masses and the lightest neutralino becomes the LSP again. The areas can be seen in Fig. 12 $\mathrm{b}$, where also the domains of $\tilde{\chi}_{2}^{0}$ decay modes exceeding $50 \%$ are marked. The thin WMAP-preferred relic density band lies along the line where the lightest neutralino can coannihilate with the lighter stop, hence reducing the relic density to an acceptable level. Here the $\widetilde{\chi}_{2}^{0}$ decays dominantly to $\chi_{1}^{0} h$ or to $\tilde{\tau}_{1} \tau$ pair, as indicated in the figure. 

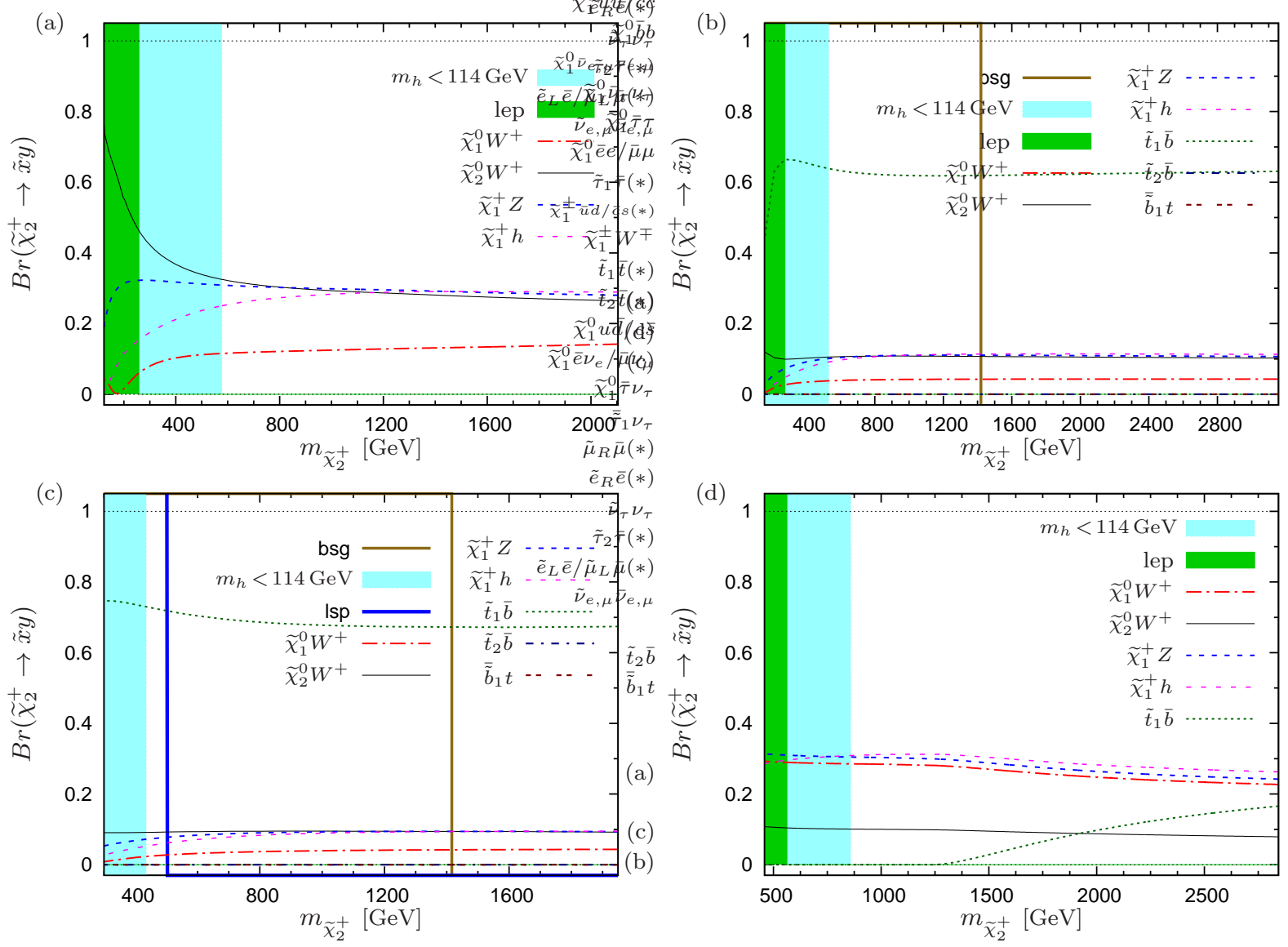

FIG. 10: Branching ratios for the decays of $\tilde{\chi}_{2}^{+}$in (a) mSUGRA model, (b) mirage mediation scenario with $\alpha=0.5$ and (c) $\alpha=1$, and (d) the AMSB scenario.

\section{SUMMARY AND DISCUSSION}

The lightest neutralino is expected to be the lightest supersymmetric particle in supersymmetric models with $R$ parity conservation. It is expected to be the end product of decays of supersymmetric partners of the Standard Model particles that are likely to be produced at the Large Hadron Collider. Thus, its mass, and its properties are of considerable importance for the supersymmetric phenomenology. The mass of the lightest neutralino, as well as those of its heavier partners, depend on the mechanism of supersymmetry breaking in the gaugino sector.

We have carried out a detailed study of the spectrum of neutralinos and charginos in different models of supersymmetry breaking gaugino masses. Because of its importance, we have investigated the properties of the lightest neutralino in different patterns of supersymmetry breaking in the gaugino sector. We have calculated lower limits on the masses of the neutralinos and the charginos, taking into account the current experimental limits on the mass of the lightest chargino. Although these limits depend, through radiative corrections, on parameters other than gaugino sector parameters, we have found that this dependence is mild, and thus the limits for the neutralino and chargino masses can be considered to be relatively robust.

We have calculated an upper bound on the mass of the lightest neutralino as a function of the lightest chargino mass. We see that for the models of supersymmetry breaking considered in this paper, only in the mirage mediation model with large $\alpha$, the upper bound found from the lower right hand two-by-two part of the mass matrix becomes relevant.

The sum rule for the neutralino and chargino squared masses is one of the distinguishing features of the supersymmetry breaking mechanism in the gaugino sector. For mSUGRA and AMSB the sign of the average squared mass difference for charginos and neutralinos is different for $M_{1}$ larger than $\mathcal{O}(100 \mathrm{GeV})$, and for mirage mediation the parameter $\alpha$ can be deduced from the sum rule.

We have also discussed in detail the decay patterns of the neutralinos and charginos in different models. An interesting result of our work is that detection of neutralino and chargino decay patterns gives important information on the 

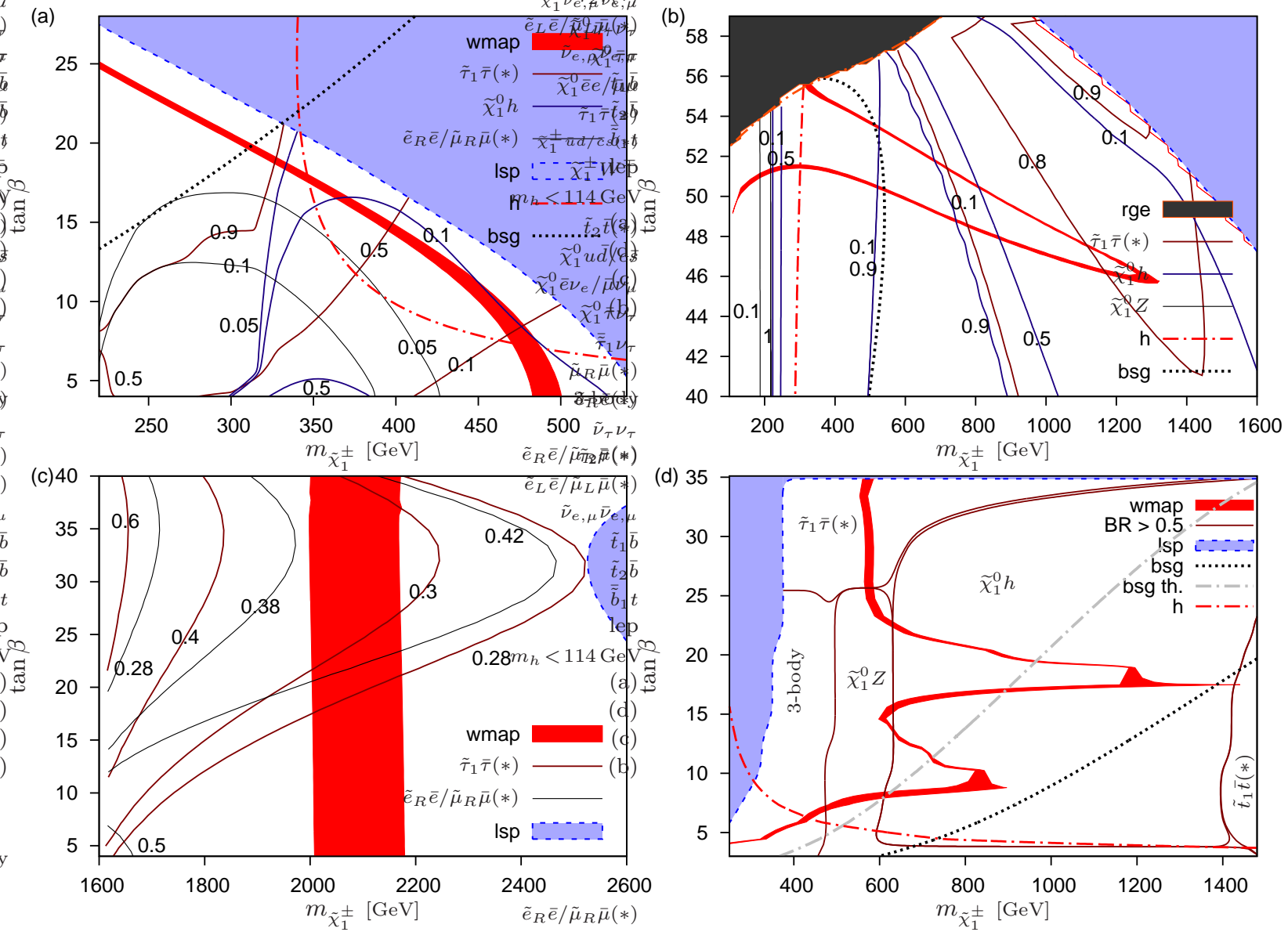

FIG. 11: Contours of constant branching ratio for the leading two-body decay modes of $\widetilde{\chi}_{2}^{0}$ superposed on the same plot with several constraints for (a) mSUGRA scenario for $m_{0}=120 \mathrm{GeV}$, (b) $m_{0}=1 \mathrm{TeV}$, (c) AMSB for $m_{0}=5 \mathrm{TeV}$ and (d) the mirage mediation for $\alpha=1$. The $b \rightarrow s \gamma$ constraint is obeyed right of the dotted bsg-denoted line and the lightest Higgs mass is more than $114 \mathrm{GeV}$ on the right of the $\mathrm{h}$ denoted dash-dotted line.

nature of the underlying supersymmetry breaking mechanism, and may help in identifying the correct supersymmetry breaking pattern.

In Section $\mathrm{V}$ it was shown that the second lightest neutralino and the lighter chargino are produced in large amounts in squark decays. This is interesting, since a promising signal to detect weakly interacting particles at Tevatron and at LHC is considered to be the associated production $\tilde{\chi}_{1}^{ \pm} \tilde{\chi}_{2}^{0}$, see e.g. 71, 72] and references therein. Let us consider produced $\tilde{\chi}_{1}^{ \pm}, \tilde{\chi}_{2}^{0}$ in view of the cascade decays in Figs. (6) -(9). It is seen that in the studied breaking patterns the largeness of the trilepton signal varies significantly. In the mSUGRA pattern, $\tilde{t}_{1}$ decays to all the heavier neutralinos and charginos with nonnegligible branching fractions. The contribution $\tilde{t}_{1} \rightarrow \tilde{\chi}_{1}^{+} b / \tilde{\chi}_{2}^{0} t$ is at a few percent level, but more events come from the decays of $\tilde{\chi}_{3,4}^{0}, \tilde{\chi}_{2}^{+}$. Thus from $\tilde{t}_{1} \overline{\tilde{t}}_{1}$ production there is an additional contribution to the trilepton signal, accompanied by a number of jets. In the AMSB pattern, the enhancement of trileptons is significant. $\tilde{t}_{1}$ 's decay $60 \%$ of the time to $\chi_{2}^{0} t$ and $20 \%$ of the time to $\chi_{1}^{+} b$. As soon as kinematically possible, the $\chi_{2}^{0}$ decays to a slepton and lepton, and $\chi_{1}^{+}$decays leptonically $25 \%$ of the time. In mirage pattern, stops tend to decay directly to the lightest neutralino and no enhancement is expected.

Since the lightest neutralino is a possible candidate for the particle dark matter, we have calculated its relic density in different supersymmetry breaking models combining the information coming from decay patterns. While in the mSUGRA model typically a narrow range with the observed relic density occurs, in the AMSB model the relic density remains below the WMAP limit for the sub-TeV scale spectrum. In mirage mediation models the observed dark matter range is narrow and close to the stop LSP region, unless the heavy Higgs resonance can be found. We note that it is not necessary that neutralino is the only dark matter particle, even if it were the lightest supersymmetric particle. Furthermore, it is possible that the R-parity is broken at least slightly in nature. This would lead to the neutralino decay, even if the breaking were so tiny that it would not show up in the experiments. 

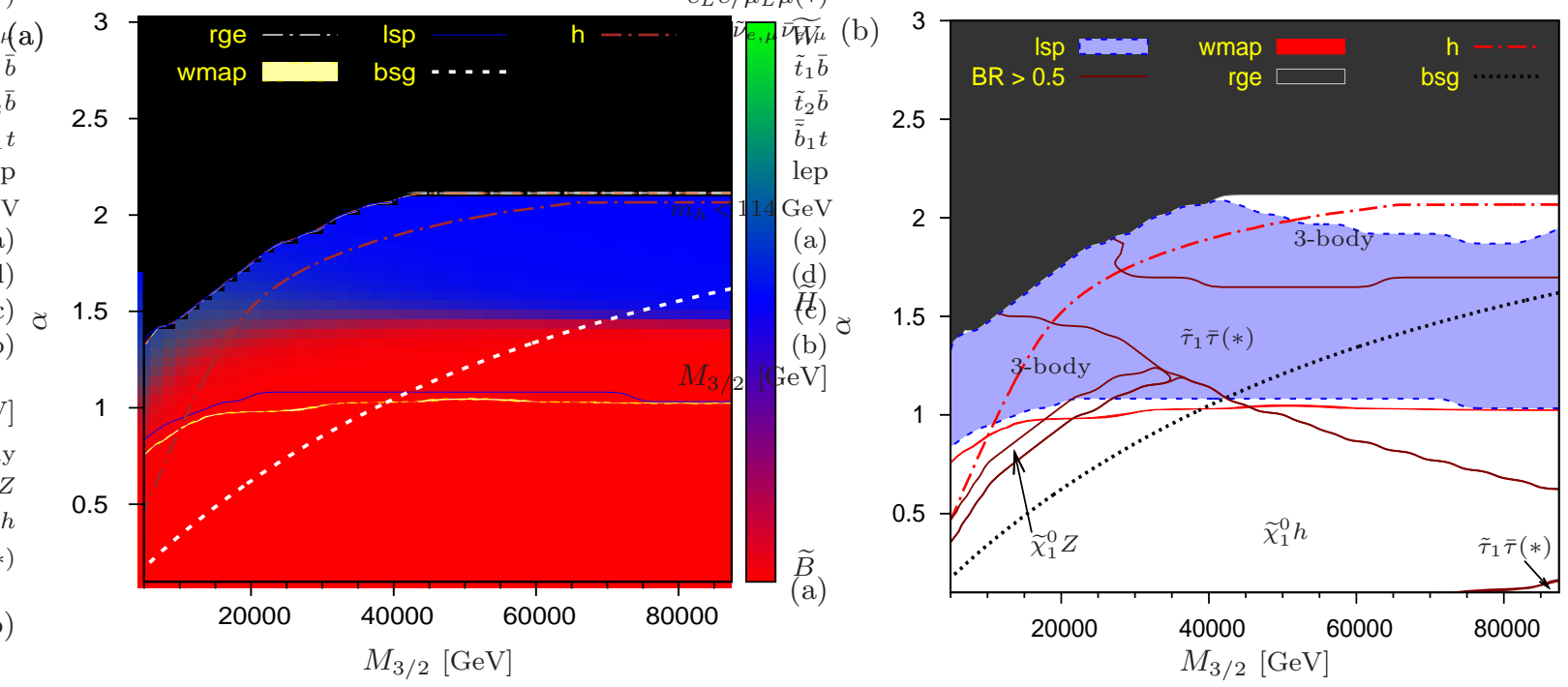

FIG. 12: Lightest neutralino composition (a) and the leading $\widetilde{\chi}_{2}^{0}$ decay modes (b) in the mirage mediation scenario in $\left(M_{3 / 2}, \alpha\right)$ plane for $\operatorname{sgn}(\mu)=+1, \tan \beta=10$ and $a_{i}=c_{i}=1$. The narrow light yellow band in (a) (red in (b)) indicates the WMAP preferred relic density area. The $b \rightarrow s \gamma$ constraint is obeyed below the dotted bsg-denoted line and the lightest Higgs mass is more than $114 \mathrm{GeV}$ below the $\mathrm{h}$ denoted dash-dotted line. The Isp denoted (light blue) line near the WMAP filling limits the area, above which the lightest neutralino is not the LSP except for the area near $\alpha=2$, which can better be seen in (b). The black areas limited by the rge-denoted line depicts the area where there are either tachyons or no REWSB. In (b) the domains of branching ratio exceeding $50 \%$ for the leading decay modes of $\widetilde{\chi}_{2}^{0}$ are drawn for the same parameters, including the constraints.

\section{ACKNOWLEDGMENTS}

KH and PT acknowledge the support by the Academy of Finland (Project No. 115 032). The work of J.L. is supported in part by the Foundation for Fundamental Research of Matter (FOM) and the Bundesministerium für Bildung und Forschung, Berlin-Bonn. The work of P.N.P. is supported by the J. C. Bose National Fellowship, the Board of Research in Nuclear Sciences, and by the Council of Scientific and Industrial Research, India. He would like to thank the Helsinki Institute of Physics, where part of this work was done, for its hospitality. PT thanks Magnus Ehrnrooth Foundation for support.

\section{Appendix A: Chargino and Neutralino Mass Matrices}

In the wino-Higgsino basis

$$
\psi_{j}^{+}=\left(-i \lambda^{+}, \psi_{H_{2}}^{1}\right), \quad \psi_{j}^{-}=\left(-i \lambda^{-}, \psi_{H_{1}}^{2}\right), \quad j=1,2,
$$

where $\lambda^{ \pm}=(1 / \sqrt{2})\left(\lambda^{1} \mp \lambda^{2}\right)$, and the superscripts 1,2 refer to $S U(2)_{L}$ indices, the chargino mass matrix can be written as [19]

$$
\mathcal{M}_{ \pm}=\left(\begin{array}{cc}
M_{2} & \sqrt{2} M_{W} \sin \beta \\
\sqrt{2} M_{W} \cos \beta & \mu
\end{array}\right),
$$

where $M_{2}$ is the supersymmetry breaking $S U(2)_{L}$ gaugino mass, $\mu$ is the Higgs(ino) mixing parameter, and $\tan \beta$ is the ratio of the vacuum expectation values of the neutral components of the two Higgs doublets $H_{2}$ and $H_{1}$. We shall denote the eigenstates of the chargino mass matrix (A2) as $\tilde{\chi}_{1}^{ \pm}$and $\tilde{\chi}_{2}^{ \pm}$, with eigenvalues $M_{\tilde{\chi}_{i=1,2}^{ \pm}}$, respectively. The eigenvalues are most easily obtained from the diagonalization of $\mathcal{M}_{ \pm}^{\dagger} \mathcal{M}_{ \pm}$resulting in the squares of the chargino masses

$$
M_{\chi_{1,2}^{ \pm}}^{2}=\frac{1}{2}\left[M_{2}^{2}+\mu^{2}+2 m_{W}^{2} \mp \sqrt{\left(M_{2}^{2}+\mu^{2}+2 m_{W}^{2}\right)^{2}-4\left(M_{2} \mu-m_{W}^{2} \sin 2 \beta\right)^{2}}\right] .
$$


On the other, in the bino-wino-Higgsino basis

$$
\psi_{j}^{0}=\left(-i \lambda^{\prime},-i \lambda^{3}, \psi_{H_{1}}^{1}, \psi_{H_{2}}^{2}\right), \quad j=1,2,3,4
$$

where $\lambda^{\prime}$ and $\lambda^{3}$ are the two-component gaugino states corresponding to the $U(1)_{Y}$ and the third component of the $S U(2)_{L}$ gauge groups, respectively, and $\psi_{H_{1}}^{1}, \psi_{H_{2}}^{2}$ are the two-component Higgsino states, the neutralino mass matrix can be written as 19]

$$
\mathcal{M}_{0}=\left(\begin{array}{cccc}
M_{1} & 0 & -M_{Z} \cos \beta \sin \theta_{W} & M_{Z} \sin \beta \sin \theta_{W} \\
0 & M_{2} & M_{Z} \cos \beta \cos \theta_{W} & -M_{Z} \sin \beta \cos \theta_{W} \\
-M_{Z} \cos \beta \sin \theta_{W} & M_{Z} \cos \beta \cos \theta_{W} & 0 & -\mu \\
M_{Z} \sin \beta \sin \theta_{W} & -M_{Z} \sin \beta \cos \theta_{W} & -\mu & 0
\end{array}\right) .
$$

$M_{1}$ is the supersymmetry breaking $U(1)_{Y}$ gaugino mass, and $g^{\prime}$ and $g$ are the gauge couplings associated with the $U(1)_{Y}$ and the $S U(2)_{L}$ gauge groups, respectively, with $\tan \theta_{W}=g^{\prime} / g$, and $M_{Z}^{2}=\left(g^{2}+g^{\prime 2}\right)\left(v_{1}^{2}+v_{2}^{2}\right) / 2$. The neutralino mass matrix can be diagonalized by a unitary transformation $N$

$$
N^{\dagger} \mathcal{M}_{0} N=\mathcal{M}_{0}^{\text {diagonal }}
$$

Assuming CP conservation, this transformation is an orthogonal transformation. We shall denote the eigenstates of the neutralino mass matrix by $\tilde{\chi}_{1}^{0}, \tilde{\chi}_{2}^{0}, \tilde{\chi}_{3}^{0}, \tilde{\chi}_{4}^{0}$ with eigenvalues $M_{\tilde{\chi}_{i=1,2,3,4}^{0}}$, labeled in order of increasing mass. Explicit expressions for these can be obtained, but these are not very illuminating. The neutralinos are mixtures of gauginos and Higgsinos

$$
\tilde{\chi}_{i}^{0}=N_{i 1} \lambda^{\prime}+N_{i 2} \lambda^{3}+N_{i 3} \psi_{H_{1}}^{1}+N_{i 4} \psi_{H_{2}}^{2} .
$$

One can obtain information on the neutralino masses by studying the expansion of the neutralino mass matrix A5 in terms of $M_{Z} / \mu$ for $M_{Z} \ll \mu$. This expansion is obtained most conveniently by using the basis $\left(-i \tilde{\gamma},-i \tilde{Z}^{0}, \tilde{H}_{a}^{0}, \tilde{H}_{b}^{0}\right)$, where

$$
\begin{aligned}
\tilde{\gamma} & =\frac{1}{\sqrt{g^{2}+g^{\prime 2}}}\left(g^{\prime} \lambda^{3}+g \lambda^{\prime}\right), \\
\tilde{Z}^{0} & =\frac{1}{\sqrt{g^{2}+g^{\prime 2}}}\left(g \lambda^{3}-g^{\prime} \lambda^{\prime}\right), \\
\tilde{H}_{a}^{0} & =\frac{1}{\sqrt{v_{1}^{2}+v_{2}^{2}}}\left(v_{1} \psi_{H_{1}}^{1}-v_{2} \psi_{H_{2}}^{2}\right), \\
\tilde{H}_{b}^{0} & =\frac{1}{\sqrt{v_{1}^{2}+v_{2}^{2}}}\left(v_{2} \psi_{H_{1}}^{1}+v_{1} \psi_{H_{2}}^{2}\right),
\end{aligned}
$$

are the photino, zino, and linear combinations of Higgsino states. In this basis, after a similarity transformation (see e.g. [8]), the neutralino mass matrix can be written as

$$
\widetilde{\mathcal{M}_{0}}=\left(\begin{array}{llll}
M_{1} & 0 & -M_{Z} \cos \left(\beta-\frac{\pi}{4}\right) s_{W} & M_{Z} \sin \left(\beta-\frac{\pi}{4}\right) s_{W} \\
0 & M_{2} & M_{Z} \cos \left(\beta-\frac{\pi}{4}\right) c_{W} & -M_{Z} \sin \left(\beta-\frac{\pi}{4}\right) c_{W} \\
-M_{Z} \cos \left(\beta-\frac{\pi}{4}\right) s_{W} & M_{Z} \cos \left(\beta-\frac{\pi}{4}\right) c_{W} & \mu & 0 \\
M_{Z} \sin \left(\beta-\frac{\pi}{4}\right) s_{W} & -M_{Z} \sin \left(\beta-\frac{\pi}{4}\right) c_{W} & 0 & -\mu
\end{array}\right) .
$$

The mass matrix A12 can be diagonalized by using perturbation theory for values of $M_{Z} \ll \mu$. For the case $M_{1}<M_{2}$, which is what one obtains in gravity mediated supersymmetry breaking (see below), the mass of the lightest neutralino can be written as, up to terms of $\mathcal{O}\left(M_{Z} / \mu\right)^{2}$,

$$
m_{\chi_{1}^{0}}=M_{1}-\frac{M_{Z}^{2} s_{W}^{2}}{\mu} \sin 2 \beta-\frac{1}{\mu^{2}}\left(M_{Z}^{2} s_{w}^{2} M_{1}+\frac{M_{Z}^{4} s_{W}^{2} c_{W}^{2}}{M_{2}-M_{1}} \sin ^{2} 2 \beta\right) .
$$

Similarly, for the second lightest neutralino $\chi_{2}^{0}$ one obtains

$$
m_{\chi_{2}^{0}}=M_{2}-\frac{M_{Z}^{2} c_{W}^{2}}{\mu} \sin 2 \beta-\frac{1}{\mu^{2}}\left(M_{Z}^{2} c_{W}^{2} M_{2}+\frac{M_{Z}^{4} s_{W}^{2} c_{W}^{2}}{M_{1}-M_{2}} \sin ^{2} 2 \beta\right)
$$


where $c_{W}^{2} \equiv \cos ^{2} \theta_{W}$ and $s_{W}^{2} \equiv \sin ^{2} \theta_{W}$. If instead we have $M_{2}<M_{1}$, a situation that arises in anomaly mediated supersymmetry breaking models, Eq. (A14) would represent the mass of the lightest neutralino $\chi_{1}^{0}$, and Eq. (A13) would give the formula for the mass of the second lightest neutralino. The dependence of the lightest neutralino mass on the specific SUSY breaking scenario is due to the fact that the ordering of the gaugino mass parameters is model dependent. The leading term in the remaining two neutralino masses is the Higgs(ino) mixing parameter $|\mu|$. Thus if $|\mu|$ value is small compared to $M_{1,2}$, Higgsino can form a large or even dominant component of the lightest neutralino, as can be seen from the mass formulae for the remaining two neutralinos:

$$
m_{\widetilde{\chi}_{3}^{0}}=\mu+\frac{M_{Z}^{2}}{2}(1+\sin 2 \beta) \frac{\mu-s_{W}^{2} M_{2}-c_{W}^{2} M_{1}}{\left(\mu-M_{1}\right)\left(\mu-M_{2}\right)}+\frac{M_{Z}^{4}}{8 \mu^{3}} \cos ^{2} 2 \beta,
$$

and

$$
m_{\widetilde{\chi}_{4}^{0}}=-\mu-\frac{M_{Z}^{2}}{2}(1-\sin 2 \beta) \frac{\mu+s_{W}^{2} M_{2}+c_{W}^{2} M_{1}}{\left(\mu+M_{1}\right)\left(\mu+M_{2}\right)}-\frac{M_{Z}^{4}}{8 \mu^{3}} \cos ^{2} 2 \beta .
$$

[1] A. Bartl, H. Fraas and W. Majerotto, Nucl. Phys. B 278, 1 (1986).

[2] A. Bartl, H. Fraas, W. Majerotto and N. Oshimo, Phys. Rev. D 40, 1594 (1989).

[3] P. N. Pandita, Phys. Rev. D 50, 571 (1994).

[4] P. N. Pandita, " Z. Phys. C 63, 659 (1994).

[5] P. N. Pandita, Phys. Rev. D 53, 566 (1996).

[6] P. N. Pandita, arXiv:hep-ph/9701411

[7] S. Y. Choi, J. Kalinowski, G. Moortgat-Pick and P. M. Zerwas, Eur. Phys. J. C 22, 563 (2001) [Addendum-ibid. C 23, 769 (2002)] arXiv:hep-ph/0108117;

[8] K. Huitu, J. Laamanen and P. N. Pandita, Phys. Rev. D 67, 115009 (2003) arXiv:hep-ph/0303262.

[9] G. Bertone, D. Hooper and J. Silk, Phys. Rept. 405, 279 (2005) arXiv:hep-ph/0404175.

[10] A. Chamseddine, R. Arnowitt, P. Nath, Phys. Rev. Lett. 49, 970 (1982); R. Barbieri, S. Ferrara, C.A. Savoy, Phys. Lett. B119, 343 (1982); H. P. Nilles, Phys. Lett. B 115, 193 (1982); Nucl. Phys. B 217, 366 (1983); H. P. Nilles, M. Srednicki and D. Wyler, Phys. Lett. B 120, 346 (1983); N. Ohta, Prog. Theor. Phys. 70, 542 (1983); L.J. Hall, J. Lykken, S. Weinberg, Phys. Rev. D27, 2359 (1983).

[11] M. Dine, A. Nelson, Phys. Rev. D48, 1277 (1993); M. Dine, A. Nelson, Y. Shirman, Phys. Rev. D51, 1362 (1995); M. Dine, A. Nelson, Y. Nir, Y. Shirman, Phys. Rev. D53, 2658 (1996).

[12] L. Randall, R. Sundrum, Nucl. Phys. B557, 79 (1999); G. Giudice, M. Luty, H. Murayama, R. Rattazzi, JHEP, 9812, 027 (1998) ; J.A. Bagger, T. Moroi, E. Poppitz, JHEP 0004, 009 (2000).

[13] S. Kachru, R. Kallosh, A. Linde and S. P. Trivedi, Phys. Rev. D 68, 046005 (2003) arXiv:hep-th/0301240.

[14] S. Kachru, M. B. Schulz and S. Trivedi, JHEP 0310, 007 (2003) arXiv:hep-th/0201028.

[15] K. Choi and H. P. Nilles, JHEP 0704 (2007) 006 arXiv:hep-ph/0702146.

[16] B. Altunkaynak, P. Grajek, M. Holmes, G. Kane and B. D. Nelson, JHEP 0904, 114 (2009) [arXiv:0901.1145 [hep-ph]].

[17] V. Lowen and H. P. Nilles, arXiv:0907.4983 [hep-ph].

[18] D. N. Spergel et al. [WMAP Collaboration], Astrophys. J. Suppl. 170 (2007) 377 arXiv:astro-ph/0603449.

[19] H. P. Nilles, Phys. Rep. 110, 1 (1984); Pran Nath, R. Arnowitt, and A. H. Chamseddine, in Supersymmetry, Supergravity and Perturbative QCD, edited by P. Roy and V. Singh (Springer, Heidelberg, 1984).

[20] LEPSUSYWG, ALEPH, DELPHI, L3 and OPAL experiments, note LEPSUSYWG/01-03.1 (http://lepsusy.web.cern.ch/lepsusy/Welcome.html).

[21] W. M. Yao et al. [Particle Data Group], J. Phys. G 33, 1 (2006).

[22] J. Abdallah et al. [DELPHI Collaboration], Eur. Phys. J. C 31, 421 (2004) arXiv:hep-ex/0311019].

[23] H. K. Dreiner, S. Heinemeyer, O. Kittel, U. Langenfeld, A. M. Weber and G. Weiglein, arXiv:0901.3485 [hep-ph].

[24] C. Cheung, A. L. Fitzpatrick and D. Shih, JHEP 0807, 054 (2008) arXiv:0710.3585 [hep-ph]]; P. Meade, N. Seiberg and D. Shih, Prog. Theor. Phys. Suppl. 177, 143 (2009) arXiv:0801.3278 [hep-ph]].

[25] L. Randall, R. Sundrum, Nucl. Phys. B557, 79 (1999).

[26] A. Pomarol, R. Rattazzi, JHEP 9905, 013 (1999).

[27] E. Katz, Y. Shadmi, Y. Shirman, JHEP 9908, 015 (1999).

[28] R. Rattazzi, A. Strumia, J.D. Wells, Nucl. Phys. B576, 3 (2000); Z. Chacko, M. Luty, E. Pontón, Y. Shadmi, Y. Shirman, hep-ph/0006047; Z. Chacko, M.A. Luty, I. Maksymsk, E. Pontón, JHEP 0004, 001 (2000).

[29] I. Jack, D.R.T. Jones, Phys. Lett. B482, 167 (2000); N. Arkani-Hamed, D.E. Kaplan, H. Murayama, Y. Nomura, JHEP 0102, 041 (2001) hep-ph/0012103.

[30] M. Carena, K. Huitu, T. Kobayashi, Nucl. Phys. B592, 164 (2001).

[31] T. Gherghetta, G. F. Giudice and J. D. Wells, Nucl. Phys. B 559, 27 (1999) arXiv:hep-ph/9904378.

[32] D.E. Kaplan, G.D. Kribs, JHEP 09, 048 (2000).

[33] K. Choi, K. S. Jeong and K. i. Okumura, JHEP 0509, 039 (2005) arXiv:hep-ph/0504037. 
[34] M. Endo, M. Yamaguchi and K. Yoshioka, Phys. Rev. D 72, 015004 (2005) arXiv:hep-ph/0504036.

[35] A. Falkowski, O. Lebedev and Y. Mambrini, JHEP 0511, 034 (2005)[arXiv: hep-ph/0507110.

[36] H. Baer, E.-K. Park, X. Tata and T. T. Wang hep-ph/0604253

[37] H. Baer, E.-K. Park, X. Tata and T. T. Wang, hep-ph/0607085.

[38] M. Endo, K. Hamaguch and F. Takahashi, Phys. Rev. Lett. 96 (2006) 211301 arXiv:hep-ph/0602061; S. Nakamura and M. Yamaguchi, Phys. Lett. B 638, 389 (2006) arXiv:hep-ph/0602081; T. Asaka, S. Nakamura and M. Yamaguchi, Phys. Rev. D74, 023520 (2006) arXiv:hep-ph/0604132; M. Dine, R. Kitano, A. Morisse and Y. Shirman, Phys. Rev. D73, 123518 (2006) arXiv:hep-ph/0604140

[39] R. Kitano and Y. Nomura, Phys. Rev. D 73, 095004 (2006) arXiv:hep-ph/0602096.

[40] K. Kawagoe and M. Nojiri, Phys. Rev. D 74, 115001 (2006) arXiv:hep-ph/0606104.

[41] K. Choi, K. Y. Lee, Y. Shimizu, Y. G. Kim and K. i. Okumura, JCAP 0612, 017 (2006) arXiv:hep-ph/0609132.

[42] W.S. Cho, Y.G. Kim, K.Y. Lee, C.B. Park and Y. Shimzu, JHEP 0704, 054 (2007) arXiv:hep-ph/0703163

[43] K. Choi, A. Falkowski, H. P. Nilles, M. Olechowski and S. Pokorski, JHEP 0411, 076 (2004) arXiv:hep-th/0411066; K. Choi, A. Falkowski, H. P. Nilles and M. Olechowski, Nucl. Phys. B718, 113 (2005) arXiv:hep-th/0503216.

[44] K. Choi, K. S. Jeong, T. Kobayashi and Ken-ichi Okumura, Phys. Lett. B 633, 355 (2006) arXiv:hep-ph/0508029]; R. Kitano and Y. Nomura, Phys. Lett. B 631, 58 (2005) arXiv:hep-ph/0509039; O. Lebedev, H. P. Nilles and M. Ratz, arXiv:hep-ph/0511320; A. Pierce and J. Thaler, arXiv:hep-ph/0604192

[45] K. Choi, K. S. Jeong and K. i. Okumura, JHEP 0509, 039 (2005) arXiv:hep-ph/0504037.

[46] B. C. Allanach, "SOFTSUSY: A C++ program for calculating supersymmetric spectra," Comput. Phys. Commun. 143, 305 (2002) arXiv:hep-ph/0104145.

[47] J. F. Gunion and H. E. Haber, Phys. Rev. D 37, 2515 (1988).

[48] A. Djouadi, M. M. Muhlleitner and M. Spira, Acta Phys. Polon. B 38, 635 (2007) arXiv:hep-ph/0609292.

[49] W. Beenakker, M. Klasen, M. Kramer, T. Plehn, M. Spira and P. M. Zerwas, Phys. Rev. Lett. 83 (1999) 3780 [Erratum-ibid. 100 (2008) 029901] arXiv:hep-ph/9906298.

[50] W. Beenakker, R. Hopker, M. Spira and P. M. Zerwas, Nucl. Phys. B 492, 51 (1997) arXiv:hep-ph/9610490; W. Beenakker, S. Brensing, M. Kramer, A. Kulesza, E. Laenen and I. Niessen, JHEP 1008, 098 (2010) [arXiv:1006.4771] [hep-ph]].

[51] M. Kamionkowski and M. S. Turner, Phys. Rev. D 42, 3310 (1990).

[52] M. Kaplinghat and M. S. Turner, Phys. Rev. Lett. 86, 385 (2001) arXiv:astro-ph/0007454.

[53] E. A. Baltz and H. Murayama, JHEP 0305, 067 (2003) arXiv:astro-ph/0108172.

[54] M. Drees and M. M. Nojiri, Phys. Rev. D 47, 376 (1993) arXiv:hep-ph/9207234.

[55] H. Baer and M. Brhlik, for Phys. Rev. D 53, 597 (1996) arXiv:hep-ph/9508321.

[56] J. Edsjo and P. Gondolo, Phys. Rev. D 56, 1879 (1997) arXiv:hep-ph/9704361.

[57] J. R. Ellis, T. Falk and K. A. Olive, Mass Phys. Lett. B 444, 367 (1998) arXiv:hep-ph/9810360.

[58] R. L. Arnowitt and B. Dutta, arXiv:hep-ph/0112157.

[59] J. R. Ellis, K. A. Olive, Y. Santoso and V. C. Spanos, Phys. Lett. B 565, 176 (2003) arXiv:hep-ph/0303043.

[60] H. Baer, C. Balazs, A. Belyaev, T. Krupovnickas and X. Tata, o s JHEP 0306, 054 (2003) arXiv:hep-ph/0304303].

[61] T. Moroi and L. Randall, Nucl. Phys. B 570, 455 (2000) arXiv:hep-ph/9906527.

[62] A. J. Barr, C. G. Lester, M. A. Parker, B. C. Allanach and P. Richardson, JHEP 0303, 045 (2003) arXiv:hep-ph/0208214.

[63] S. Profumo and P. Ullio, arXiv:astro-ph/0404390

[64] D. Hooper and L. T. Wang, Phys. Rev. D 69, 035001 (2004) arXiv:hep-ph/0309036.

[65] M. Nagai and K. Nakayama, Phys. Rev. D 76, 123501 (2007) [arXiv:0709.3918 [hep-ph]].

[66] H. Abe, Y. G. Kim, T. Kobayashi and Y. Shimizu, JHEP 0709, 107 (2007) arXiv:0706.4349 [hep-ph]].

[67] H. Baer, E. K. Park, X. Tata and T. T. Wang, n," JHEP 0706, 033 (2007) arXiv:hep-ph/0703024.

[68] G. Belanger, F. Boudjema, A. Pukhov and A. Semenov, in Comput. Phys. Commun. 176, 367 (2007) arXiv:hep-ph/0607059.

[69] E. Barberio et al. [Heavy Flavor Averaging Group (HFAG) Collaboration], arXiv:0704.3575 [hep-ex].

[70] H. Baer, A. Mustafayev, E. K. Park and X. Tata, JHEP 0805 (2008) 058 arXiv:0802.3384 [hep-ph]].

[71] V. D. Barger and C. Kao, Phys. Rev. D 60, 115015 (1999) arXiv:hep-ph/9811489.

[72] C. S. Li, Z. Li, R. J. Oakes and L. L. Yang, Phys. Rev. D 77, 034010 (2008) [arXiv:0707.3952 [hep-ph]]. 